UCI-04-43

May 2004

\title{
Exact Bianchi Identity in Regge Gravity
}

\author{
Herbert W. Hamber ${ }^{1}$ and Geoff Kagel ${ }^{2}$ \\ Department of Physics and Astronomy \\ University of California \\ Irvine, CA 92697-4575, USA
}

\begin{abstract}
In the continuum the Bianchi identity implies a relationship between different components of the curvature tensor, thus ensuring the internal consistency of the gravitational field equations. In this paper the exact form for the Bianchi identity in Regge's discrete formulation of gravity is derived, by considering appropriate products of rotation matrices constructed around null-homotopic paths. The discrete Bianchi identity implies an algebraic relationship between deficit angles belonging to neighboring hinges. As in the continuum, the derived identity is valid for arbitrarily curved manifolds without a restriction to the weak field small curvature limit, but is in general not linear in the curvatures.
\end{abstract}

(Submitted to Class. and Quant. Gravity)

\footnotetext{
${ }^{1}$ e-mail address : hhamber@uci.edu

${ }^{2}$ e-mail address : gkagel@uci.edu
} 


\section{Introduction}

In this paper we investigate the form of the Bianchi identities in Regge's [1] lattice formulation of gravity $[2,3,4,5,6,7,8,9,10]$. The Bianchi identities play an important role in the continuum formulation of gravity, both classical and quantum-mechanical, giving rise to a differential relationship between different components of the curvature tensor. It is known that these simply follow from the definition of the Riemann tensor in terms of the affine connection and the metric components, and help ensure the consistency of the gravitational field equations in the presence of matter. At the same time they can be regarded as a direct consequence of the local gauge (diffeomorphism) invariance of the gravitational action, since they can be derived by invoking the invariance of the action under infinitesimal gauge transformations (see for example [18, 19, 20, 21]).

In this paper we will show that the lattice formulation of gravity has an equivalent form of the Bianchi identities, which are both exact, in the sense that they are valid for arbitrarily curved lattices, and reduce to their continuum counterparts in the weak field limit. We will derive the exact lattice Bianchi identities in three (Section 4) and four dimensions (Section 5) explicitly by considering the product of rotation matrices along paths which are topologically trivial (i.e. reducible to a point). By expressing the rotation matrices about each hinge in terms of the local representatives of the curvatures, namely the deficit angles, we will obtain an algebraic relationship between deficit angles, area and volumes pertaining to neighboring simplices.

For lattices which are close to flat, it will be shown that the derived set of identities is analogous to the Bianchi identities in the continuum, once the edge lengths are identified with appropriate components of the metric in the continuum (Sections 9 and 10). We will therefore extend and complete previous results on the lattice Bianchi identities, which so far have been restricted to the weak field limit $[1,3,4,22,23]$. The results presented in this paper should therefore be relevant for both classical (see for example [24, 25, 26, 27] an references therein) and quantum (see for example $[28,29,30,31,32,33,34,35,36,37,38]$ and references therein) discrete gravity. ${ }^{3}$

It is well known that in the continuum the Bianchi identity for the curvature tensor ensures the consistency of the Einstein field equations. For the Riemann curvature tensor the un-contracted Bianchi identities read

$$
R_{\nu \alpha \beta ; \gamma}^{\mu}+R_{\nu \gamma \alpha ; \beta}^{\mu}+R_{\nu \beta \gamma ; \alpha}^{\mu}=0
$$

\footnotetext{
${ }^{3}$ Recent reviews of Regge gravity can be found in $[10,12,13,14,15,16]$, while a comprehensive collection of up-todate references is assembled in [17]. Some further mathematical aspects of piecewise linear spaces, with some relevance to lattice gravity, are discussed in the above cited references $[5,6]$, as well as in $[39,40,41,42,43,44,45,46,47]$ and references therein, and more recently in $[48,49,50,51,52,53]$ and references therein.
} 
or more concisely

$$
R_{\nu[\alpha \beta ; \gamma]}^{\mu}=0
$$

where $[\cdots]$ denotes symmetrization. These identities are easily derived by inserting into the above expression the explicit definition for the curvature tensor in terms of the metric $g_{\mu \nu}$,

$$
R_{\lambda \mu \nu \kappa}=\frac{1}{2} \frac{\partial^{2} g_{\lambda \nu}}{\partial x^{\kappa} \partial x^{\mu}}+\cdots
$$

It is then easy to see that in $d$ dimensions there are

$$
\frac{d(d-1)}{2}\left(\begin{array}{l}
d \\
3
\end{array}\right)=\frac{d^{2}(d-1)(d-2)}{12}
$$

un-contracted Bianchi identities, and thus 3 identities in $d=3$ and 24 identities in $d=4$. In their contracted form, the Bianchi identities imply for the Ricci tensor

$$
R_{\nu \alpha ; \gamma}-R_{\nu \gamma ; \alpha}+R_{\nu \gamma \alpha ; \mu}^{\mu}=0
$$

and for the scalar curvature

$$
R_{; \gamma}-2 R_{\gamma ; \mu}^{\mu}=0
$$

These relations in turn give the contracted Bianchi identity

$$
\left[R^{\mu}{ }_{\nu}-\frac{1}{2} \delta^{\mu}{ }_{\nu} R\right]_{; \mu}=0
$$

which always corresponds to $d$ equations in $d$ dimensions. We note here that a simple physical interpretation for the Bianchi identity can be given in terms of a divergence of suitably defined stresses [18]. Thus for example in three dimensions one has

$$
P_{\nu i ; i}^{\mu}=0 \quad \text { with } \quad P_{\nu i}^{\mu} \equiv \epsilon_{i j k} R_{\nu j k}^{\mu} .
$$

It is also well known that the Bianchi identities are required for ensuring the consistency of the gravitational field equations. Consider the classical field equations with a cosmological constant term,

$$
R_{\mu \nu}-\frac{1}{2} g_{\mu \nu} R+\Lambda g_{\mu \nu}=8 \pi G T_{\mu \nu}
$$

with $\Lambda=8 \pi G \lambda$ the cosmological constant. Applying a covariant derivative on both sides one has

$$
\left[R^{\mu}{ }_{\nu}-\frac{1}{2} \delta^{\mu}{ }_{\nu} R\right]_{; \mu}=8 \pi G T_{\nu ; \mu}^{\mu}
$$

which for a covariantly conserved energy-momentum tensor

$$
T_{\nu ; \mu}^{\mu}=0
$$


is only consistent if the contracted Bianchi identity of Eq. (1.7) is identically satisfied.

In $d$ dimensions one has $d$ contracted Bianchi identities. Since there are in general $d(d+1) / 2$ equations of motion, as well as $d$ harmonic gauge fixing conditions, one has for the number of independent gravitational degrees of freedom in $d$ dimensions

$$
\frac{d(d+1)}{2}-d-d=\frac{d(d-3)}{2}
$$

which indeed reproduces correctly in four dimensions the two independent helicity states appropriate for a massless spin two particle.

There exists also a close relationship between the Bianchi identity and the gauge invariance of the gravitational action. We shall take note here of the fact that the Bianchi identity can be derived from the requirement that the gravitational action

$$
I_{G}[g]=-\frac{1}{16 \pi G} \int d^{4} x \sqrt{g(x)} R(x)
$$

being a scalar, should be invariant under infinitesimal local gauge transformations at a space-time point $x$,

$$
\delta g_{\mu \nu}(x)=-g_{\mu \lambda}(x) \partial_{\nu} \chi^{\lambda}(x)-g_{\lambda \nu}(x) \partial_{\mu} \chi^{\lambda}(x)-\partial_{\lambda} g_{\mu \nu}(x) \chi^{\lambda}(x)
$$

After substituting the above expression for a gauge deformation into the variation of the action given in Eq. (1.13),

$$
\delta I_{G}[g]=\frac{1}{16 \pi G} \int d^{4} x \sqrt{g(x)}\left[R^{\mu \nu}-\frac{1}{2} g^{\mu \nu} R\right] \delta g_{\mu \nu}
$$

one obtains again, after integrating by parts, the contracted Bianchi identity of Eq. (1.7).

Let us now turn to the lattice theory. In a $d$-dimensional piecewise linear space-time the expression analogous to the Einstein-Hilbert action was given by Regge [1] as

$$
I_{R}=\sum_{\text {hinges h }} A_{h}^{(d-2)} \delta_{h}
$$

where $A_{h}^{(d-2)}$ is the volume of the hinge and $\delta_{h}$ is the deficit angle there. The above lattice action is supposed to be equivalent to the continuum expression

$$
I_{E}=\frac{1}{2} \int d^{d} x \sqrt{g} R
$$

and indeed it has been shown $[3,5,11]$ that $I_{R}$ tends to the continuum expression as the simplicial block size (or some suitable average edge length) tends to zero in the appropriate way. The above 
Regge form for the lattice action can be naturally extended to include cosmological and curvature squared terms $[7,10]$

$$
I\left(l^{2}\right)=\sum_{h}\left[\lambda V_{h}-k A_{h} \delta_{h}+a \delta_{h}^{2} A_{h}^{2} / V_{h}+\cdots\right]
$$

with $k^{-1}=8 \pi G$. In the limit of small fluctuations around a smooth background, $I\left(l^{2}\right)$ corresponds to the continuum action

$$
I[g]=\int d^{4} x \sqrt{g}\left[\lambda-\frac{k}{2} R+\frac{a}{4} R_{\mu \nu \rho \sigma} R^{\mu \nu \rho \sigma}+\cdots\right] .
$$

In the following we will focus on the Regge term (proportional to $k$ ) only.

Variations of $I_{R}$ in Eq. (1.16) with respect to the edge lengths then give the simplicial analogs of Einstein's equations, whose derivation is significantly simplified by the fact that the variation of the deficit angle is known to be zero in any dimensions,

$$
\delta I_{R}=\sum_{h} \delta\left(A_{h}^{(d-2)}\right) \delta_{h}
$$

as happens in the continuum as well (where one finds that the variation of the curvature reduces to a total derivative). In three dimensions the above action gives for the equation of motion $\delta_{h}=0$ for every hinge in the lattice, whereas in four dimensions variation with respect to $l_{p}$ yields $[1]$

$$
\frac{1}{2} l_{p} \sum_{h \supset l_{p}} \delta_{h} \cot \theta_{p h}=0
$$

where the sum is over hinges (triangles in four dimensions) labeled by $h$ meeting on the common edge $p$, and $\theta_{p h}$ is the angle in the hinge $h$ opposite to the edge $p$. The above equation is the lattice analog of the field equations of Eq. (1.9), for pure gravity and vanishing cosmological constant.

Numerical solutions to the lattice equations of motion can in general be found by appropriately adjusting the edge lengths according to Eq. (1.21). Since the resulting equations are non-linear in the edge lengths, slight complications can arise such as the existence of multiple solutions, although for sufficiently weak fields one would expect the same level of degeneracies as in the continuum [3]. Several authors have discussed the application of the Regge equations to strong field problems in classical general relativity, and some samples can be found in $[24,25,26]$. The relevance of the Bianchi identities to a numerical solution to the lattice field equations - using for example a $3+1$ time evolution scheme - resides in the fact that they are in principle a powerful tool to check the overall accuracy and consistency of the numerical solutions.

The Bianchi identities also play an important role in the quantum formulation. In a quantummechanical theory of gravity the starting point is a suitable definition of the discrete Feynman path 
integral $[8,7,10]$. In the simplicial lattice approach one starts from the discretized Euclidean Feynman path integral for pure gravity, with the squared edge lengths taken as fundamental variables,

$$
Z_{L}=\int_{0}^{\infty} \prod_{s}\left(V_{d}(s)\right)^{\sigma} \prod_{i j} d l_{i j}^{2} \Theta\left[l_{i j}^{2}\right] \exp \left\{-\sum_{h}\left(\lambda V_{h}-k \delta_{h} A_{h}+a \delta_{h}^{2} A_{h}^{2} / V_{h}+\cdots\right)\right\} \text {. }
$$

The above regularized lattice expression should be compared to the continuum Euclidean path integral for pure gravity

$$
Z_{C}=\int \prod_{x}(\sqrt{g(x)})^{\sigma} \prod_{\mu \geq \nu} d g_{\mu \nu}(x) \exp \left\{-\int d^{4} x \sqrt{g}\left(\lambda-\frac{k}{2} R+\frac{a}{4} R_{\mu \nu \rho \sigma} R^{\mu \nu \rho \sigma}+\cdots\right)\right\} .
$$

In the discrete case the integration over metrics is replaced by integrals over the elementary lattice degrees of freedom, the squared edge lengths. The discrete gravitational measure in $Z_{L}$ can be considered as the lattice analog of the DeWitt continuum functional measure $[5,6,8,9,12,38]$. A cosmological constant term is needed for convergence of the path integral, while the curvature squared term allows one to control the fluctuations in the curvature $[7,10]$. In the end one is mostly interested in the limit $a \rightarrow 0$, where the theory, in the absence of matter and after a suitable rescaling of the metric, only depends on one bare parameter, the dimensionless coupling $k^{2} / \lambda$.

In the quantum theory the Bianchi identities of Eq. (1.7) are still satisfied as operator equations, and ensure the consistency of the quantum equations of motions. In ordinary lattice nonabelian gauge theories an attempt has been made to entirely replace the functional integration over the gauge fields by an integration over field strengths, but now subject to the Bianchi identity constraint [54]. In the case of gravitation such an approach is more difficult, since the analog of the gauge field is represented by the affine connection, and not the by the curvature tensor.

\section{Lattice Parallel Transport}

To construct the lattice Bianchi identities we will follow a strategy similar to the one used in the derivation of the exact lattice Bianchi identities in non-abelian lattice gauge theories. There the Bianchi identities can be obtained by considering the path-ordered product of $S U(n)$ gauge group rotation matrices, taken along a suitable closed path encircling a cube. The path has to be chosen topologically trivial, in the sense that it can be shrunk to a point without entangling any plaquettes $[54,55]$.

Let us therefore first review the notion of parallel transport of a test vector around a small loop embedded in the lattice. Consider a closed path $\Gamma$ encircling a hinge $h$ and passing through each 
of the simplices that meet at that hinge. In particular one may take $\Gamma$ to be the boundary of the polyhedral dual area surrounding the hinge. For each neighboring pair of simplices $j, j+1$, one can write down a Lorentz transformation $L_{\mu}^{\nu}$, which describes how a given vector $\phi_{\mu}$ transforms between the local coordinate systems in these two simplices,

$$
\phi_{\mu}^{\prime}=[L(j, j+1)]_{\mu}^{\nu} \phi_{\nu}
$$

Now in general it is possible to choose coordinates so that $L_{\mu}^{\nu}$ is the identity matrix for one pair of simplices, but then it will not be unity for other pairs. The above Lorentz transformation is directly related to the continuum path-ordered $(P)$ exponential of the integral of the affine connection $\left(\Gamma_{\lambda}\right)_{\mu}^{\nu}=\Gamma_{\mu \lambda}^{\nu}$ by

$$
L_{\mu}^{\nu}=\left[P e^{\iint_{\text {between simplices }}^{\text {path }} \Gamma_{\lambda} d x^{\lambda}}\right]_{\mu}^{\nu}
$$

The connection here is intended to only have support on the common interface between the two simplices.

Next we will consider the product of rotation matrices along a closed loop $\Gamma$. The path can entangle several hinges, or just one, in which case it will be called a closed elementary loop. On the lattice the effect of parallel transport around a closed elementary loop $\Gamma$ is obtained from the matrix [7]

$$
\left[\prod_{j} L(j, j+1)\right]_{\mu \nu}=\left[e^{\delta_{h} U_{\cdot \cdot}^{(h)}}\right]_{\mu \nu}
$$

where $U_{\mu \nu}^{(h)}$ is a bivector orthogonal to the hinge $h$, defined in four dimensions by

$$
U_{\mu \nu}^{(h)}=\frac{1}{2 A_{h}} \epsilon_{\mu \nu \rho \sigma} l_{(a)}^{\rho} l_{(b)}^{\sigma}
$$

with $l_{(a)}^{\rho}$ and $l_{(b)}^{\rho}$ two vectors forming two sides of the hinge $h$. We note that in general the validity of the lattice parallel transport formula given above is not restricted to small deficit angles. For a closed path $\Gamma$, the total change in a vector $\phi_{\mu}$ which undergoes parallel transport around the path is given by

$$
\phi_{\mu}^{\prime}=\phi_{\mu}+\delta \phi_{\mu}=\left[\prod_{\substack{\text { pairs of } \\ \text { simplices on } \Gamma}} L(j, j+1)\right]_{\mu}^{\nu} \phi_{\nu}
$$

For smooth enough manifolds, the product of Lorentz transformations around a closed elementary loop $\Gamma$ can be deduced from the components of the Riemann tensor,

$$
\left[\prod_{\substack{\text { pairs of } \\ \text { simplices on } \Gamma}} L(j, j+1)\right]_{\mu}^{\nu} \approx\left[e^{R_{. \rho \sigma} \Sigma^{\rho \sigma}}\right]_{\mu}^{\nu}
$$


where $\left(R_{. \rho \sigma}\right)_{\mu}^{\nu}=R_{\mu \rho \sigma}^{\nu}$ is the curvature tensor and $\Sigma^{\rho \sigma}$ is a bivector in the plane of $\Gamma$, with magnitude equal to $1 / 2$ times the area of the loop $\Gamma$. (For a parallelogram with edges $a^{\rho}$ and $b^{\rho}$, $\left.\Sigma^{\rho \sigma}=\frac{1}{2}\left(a^{\sigma} b^{\rho}-a^{\rho} b^{\sigma}\right)\right)$. The above result then reproduces to lowest order the parallel transport formula

$$
\delta \phi_{\mu}=R_{\mu \rho \sigma}^{\nu} \Sigma^{\rho \sigma} \phi_{\nu}
$$

Comparison of Eq. (2.3) and Eq. (2.6) means that for one hinge one may make the identification

$$
R_{\mu \nu \rho \sigma} \Sigma^{\rho \sigma} \rightarrow \delta_{h} U_{\mu \nu}^{(h)}
$$

It is important to notice that this relation does not give complete information about the Riemann tensor, but only about its projection in the plane of the loop $\Gamma$, orthogonal to the given hinge. Indeed the deficit angle divided by the area of the loop can be taken as a definition of the local sectional curvature $K_{h}[10]$

$$
\frac{\delta_{h}}{A_{\Gamma_{h}}}=K_{h}=\frac{R_{\mu \nu \rho \sigma} e_{a}^{\mu} e_{b}^{\nu} e_{a}^{\rho} e_{b}^{\sigma}}{\left(g_{\mu \rho} g_{\nu \sigma}-g_{\mu \sigma} g_{\nu \rho}\right) e_{a}^{\mu} e_{b}^{\nu} e_{a}^{\rho} e_{b}^{\sigma}}
$$

which represents the projection of the Riemann curvature in the direction of the bivector $\mathbf{e}_{\mathbf{a}} \wedge \mathbf{e}_{\mathbf{b}}$.

The lattice Bianchi identities are derived by considering closed paths that can be shrunk to a point without entangling any hinge. The product of rotation matrices associated with the path then has to give the identity matrix $[1,3]$. Thus, for example, the ordered product of rotation matrices associated with the triangles meeting on a given edge has to give one, since a path can be constructed which sequentially encircles all the triangles and is topologically trivial

$$
\prod_{\substack{\text { hinges h } \\ \text { meeting on edge p }}}\left[e^{\delta_{h} U_{\cdot}^{(h)}}\right]_{\mu \nu}=1 .
$$

Other identities might be derived by considering paths that encircle hinges meeting on one point.

\section{Geometric Setup}

The discrete analogue of the Bianchi identity will be derived by considering a product of rotation matrices along a homotypically trivial path. This section discusses the general geometric setups needed to define correctly the product of rotation matrices entering the exact lattice Bianchi identities derived later in the paper (Sections 4 and 5). For the 3-d case, consider a tetrahedron with a point in its interior. Connect the vertices of the tetrahedron to the point in the center. We 
now have formed 4 tetrahedra from the original tetrahedron. In three dimensions, hinges are edges, so here we have enclosed four hinges: one connecting each vertex of the original tetrahedra to the interi

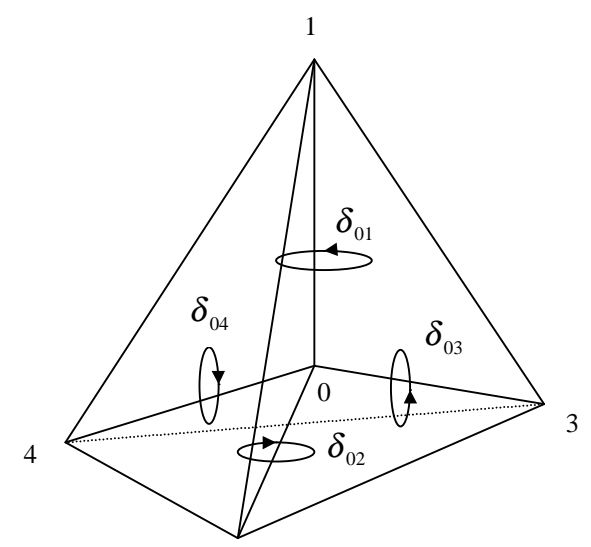

2

Fig. 1. In three dimensions four tetrahedra meet on a point, labeled here by 0 . Deficit angles $\delta_{01}, \delta_{02}, \delta_{03}$ and $\delta_{04}$ are associated with edges $0-1,0-2,0-3$ and $0-4$ respectively. The Bianchi identities are obtained by taking an ordered product of rotation matrices along a path which encircles all four hinges (edges here) and is topologically trivial, in the sense that it can be shrunk to a point.

Referring to Fig. 1, for the moment only considering flat space, call the interior point 0 (zero) and place it at the origin. Let us take our coordinate system so that vertex 1 lies on the positive $\mathrm{z}$ axis $(z 1>0)$. Let us take vertex 2 to lie in the $\mathrm{x}-\mathrm{z}$ plane with $z 2<0$ and $x 2>0$. Let us take vertex 3 to have $z 3<0, x 3<0$ and $y 3>0$. Finally, let us take vertex 4 to have $z 4<0, x 4<0$ and $y 4<0$. So, to summarize: 


$$
\begin{array}{ccccc}
\text { Vertex : } & 1 & 2 & 3 & 4 \\
z: & + & - & - & - \\
x: & 0 & + & - & - \\
y: & 0 & 0 & + & -
\end{array}
$$

Now, the following argument will apply for all cases where we have the center point completely surrounded; the aforementioned restrictions in Eq. (3.1) are mentioned only to give the reader a nice picture of the situation.

Now, "curve the space". This is done by changing one of the edge lengths. Any 9 of the 10 edge lengths can be chosen arbitrarily (provided the center point is completely surrounded by the constructed volumes and provided that real areas and real volumes are still formed) and the space will still be flat. So curvature, in this setup, just amounts to adjusting one edge length $\left(l_{34}\right.$, the edge between vertices 3 and 4, is the easiest one to adjust). For an arbitrary setup, one adds more edges until the relative flat space locations of all vertices are specified, making sure not to add any edges between points whose relative flat space location is already determined but rather marking each such edge as an "unadded edge" (and making sure that all areas and volumes are real); then, all remaining ("unadded") edges are determined for flat space, and it is the varying of those remaining edges which is the source of all curvature for that setup. This last comment applies to d-dimensions and general lattices, not just d-simplices surrounding one point, and addresses the question of curvature invariance under edge variation, i.e. curvature invariance under the edge variation of the added edges (with the "unadded" edges still unadded).

Further, let us label the four tetrahedra by a point in each of them along the line const $\times\left(\mathbf{l}_{1}+\right.$ $\mathbf{l}_{2}+\mathbf{l}_{3}$ ) where $\mathbf{l}_{1}, \mathbf{l}_{2}$ and $\mathbf{l}_{3}$ are vectors based at the point 0 , the interior point. 


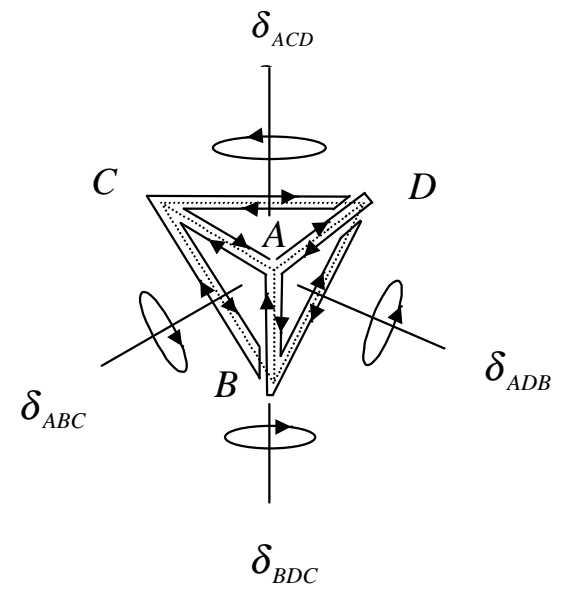

Fig. 2. A topologically trivial path which encircles four hinges in three dimensions, and can be shrunk to a point. The vertices $A, B, C$ and $D$ reside in the dual lattice, and can conveniently be placed at the centers of the tetrahedra shown in Fig. 1. It is then advantageous to label the deficit angles by the vertices in the dual lattice. The path ordered product of rotation matrices around the shown path then reduces to the identity matrix.

Referring to Fig. 1, and Fig. 2 which, with triangle BCD behind A, views Fig. 1 from underneath, let us call the point in the center of the tetrahedra formed via vertices 0123, and not 4, point $\mathrm{D}$; similarly call the point at the center of 0234 , and not 1 , point $\mathrm{A}$, that at the center of 0134, and not 2, point B and that at the center of 0124, and not 3, point C. We also have tetrahedron $\mathrm{A}$ which is the tetrahedron containing point $\mathrm{A}$, tetrahedron $\mathrm{B}$ containing point $\mathrm{B}$, etc. These four points, point A, point B, point $\mathrm{C}$ and point $\mathrm{D}$ form a Voronoi tetrahedra. Going from $A \rightarrow B \rightarrow C \rightarrow A$, for example, goes around hinge 04. In general

$\begin{array}{ccccc}\text { hinge gone around } & \text { rotation } & \text { Voronoi vertex not in path } & \text { positive path } & \text { negative path } \\ 01 & R 1 & A & B \rightarrow C \rightarrow D \rightarrow B \quad B \rightarrow D \rightarrow C \rightarrow B \\ 02 & R 2 & B & A \rightarrow D \rightarrow C \rightarrow A \quad A \rightarrow C \rightarrow D \rightarrow A \\ 03 & R 3 & C & A \rightarrow B \rightarrow D \rightarrow A \quad A \rightarrow D \rightarrow B \rightarrow A \\ 04 & R 4 & D & A \rightarrow C \rightarrow B \rightarrow A \quad A \rightarrow B \rightarrow C \rightarrow A\end{array}$

so that rotations which are clockwise when viewed from outside our setup, or counterclockwise when viewed from the point in the middle, are associated with a "-R", and rotations which are counter-clockwise when viewed from outside our setup, or clockwise when viewed from the point in the middle, are associated with a " $+\mathrm{R}$ ", as is traditional for a right handed coordinate system inside the set-up. 


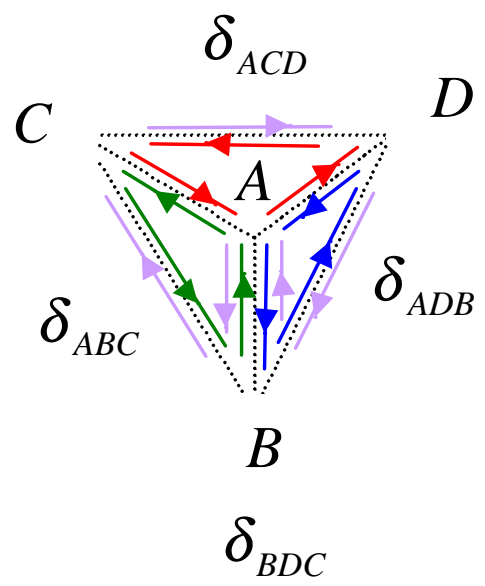

Fig. 3. Paths associated with different hinges in three dimensions are shown in different shades of gray. The product of rotation matrices around a single hinge is simply elated to the deficit angle for that hinge. In order to obtain a closed path, segment $A B$ has to be transversed more than two times.

Now, let us try and compose a null path around all four hinges. Referring to Fig. 3, we write our null path as

$$
A \rightarrow B \rightarrow D \rightarrow A, A \rightarrow D \rightarrow C \rightarrow A, A \rightarrow C \rightarrow B \rightarrow A, A \rightarrow B, B \rightarrow C \rightarrow D \rightarrow B, B \rightarrow A
$$

or, noting that the path is a null path, we have

$$
1=R_{t o t}=\Gamma_{A \rightarrow B}^{-1} R 1_{B} \Gamma_{A \rightarrow B} R 4 R 2 R 3
$$

where $\Gamma_{A \rightarrow B}$ is a rotation matrix representing the rotation that occurs when a vector is parallel transported from tetrahedron A directly to tetrahedron $\mathrm{B}, R_{t o t}$ means the total rotation matrix after going through the whole path, and $R 1_{B}$ is $R 1$ written in B's coordinate system; the other rotations, with no subscripts, are written in A's coordinate system. Eq. (3.3) can be written completely in A's coordinate system as

$$
1=R_{t o t}=R 1 R 4 R 2 R 3
$$

Also in reference to the $\Gamma$ 's, there is a subtlety to note. The $\Gamma$ 's are always "direct" $\Gamma$ 's between adjacent d-simplices. For example, $\Gamma_{A \rightarrow B}$ is not $\Gamma_{A \rightarrow C \rightarrow B}$ (the latter being starting at A, then go directly to $\mathrm{C}$, and then go directly to $\mathrm{B}$ ); indeed, since

$$
R 1=\Gamma_{A \rightarrow B} \Gamma_{A \rightarrow C \rightarrow B}^{-1}
$$


both cannot simultaneously be set equal to the unit matrix.

In general, when setting up a d-dimensional global coordinate system, one specifies the coordinate system in one d-simplex and $N_{d}-1 \Gamma$ 's $\left(N_{d}\right.$ is the number of d-simplices in the lattice) such that all d-simplices are linked directly or indirectly; to specify any more $\Gamma$ 's would be to specify a pre-determined (via edge lengths) loop rotation. One can, for e.g., choose all $N_{d}-1 \Gamma$ 's equal to the unit matrix, so that $\Gamma_{A \rightarrow B}$ in Eq. (3.4) would be one of three $\Gamma$ 's set equal to the unit matrix.

If one only specifies $\Gamma$ 's between adjacent d-simplices, one can think of this coordinate system in flat d-space as d-simplices which 1) are attached to each other only by the (d-1)-simplex "faces" corresponding to the $N_{d}-1 \Gamma$ 's, and 2) can go through each other (for e.g., consider a hinge with greater than $2 \pi$ radians around it) . Of course, when viewed in the actual curved space, the dsimplices do not go though each other. Some sample coordinate systems would be obtained by 1) specifying $\Gamma$ 's between one specific tetrahedron (or d-simplex) and every other one, such as $\Gamma_{A \rightarrow B}$, $\Gamma_{A \rightarrow C}$ and $\Gamma_{A \rightarrow D}$ in our particular set up, and 2) specifying the $\Gamma$ 's in a chainlike fashion, such as $\Gamma_{A \rightarrow B}, \Gamma_{B \rightarrow C}$, and $\Gamma_{C \rightarrow D}$ in our set up.

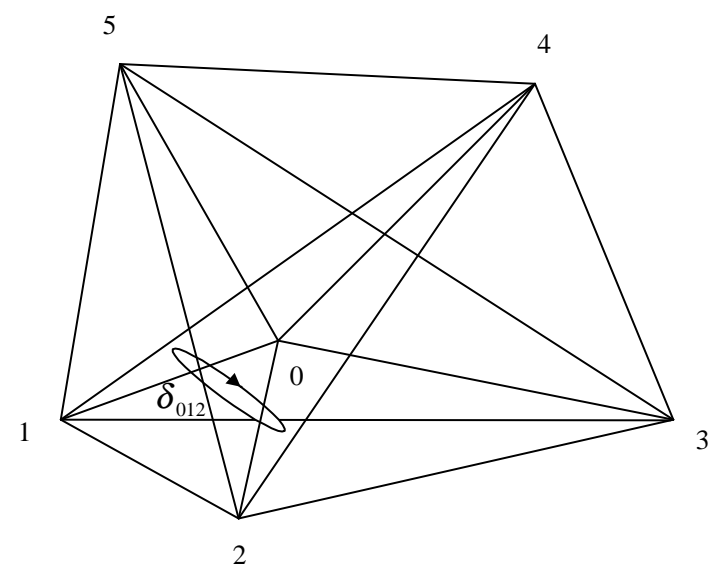


Fig. 4. Similar to Fig. 1., but now in four dimensions. In four dimensions five simplices meet on a point, labeled here by 0 . Deficit angles $\delta_{012}$ etc. are associated with triangles $0-1-2$ etc. respectively. The Bianchi identities are obtained by taking an ordered product of rotation matrices along a path which encircles several hinges (triangles here) and is topologically trivial, in the sense that it can be shrunk to a point.

Now, let us consider one four-dimensional set up in particular. Consider a four simplex with a point in the middle. This divides the original 4-simplex into 5 new 4 -simplices. Then, readjust the edge lengths and curve the space (see Fig. 4). We now consider parallel transporting the vector around a null path within this simplicial complex. Label each of the new 4-simplices A, B, C, D and E (see Fig. 5).

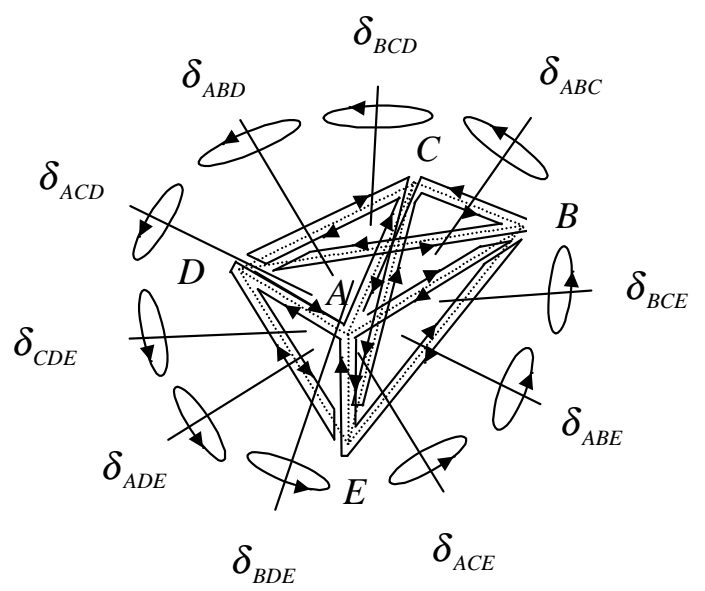

Fig. 5. Similar to Fig. 2., but now in four dimensions. The topologically trivial path which encircles all shown hinges can be shrunk to a point. The vertices $A, B, C, D$ and $E$ reside in the dual lattice, and can conveniently be placed at the centers of the simplices shown in Fig. 4.. As in the three-dimensional case, it is advantageous to label the deficit angles by the vertices in the dual lattice. The ordered product of rotation matrices around the shown path then reduces to the identity matrix.

We now make an important point for this particular set up: the number of 4-simplices around 
a triangle hinge is 3 . There are 3 vertices in the two dimensional space of the hinge and three vertices in the remaining two dimensions. The three vertices in the hinge and any two of the three vertices outside the hinge form a 4 -simplex, so that three 4 -simplices surround the hinge. ${ }^{4}$

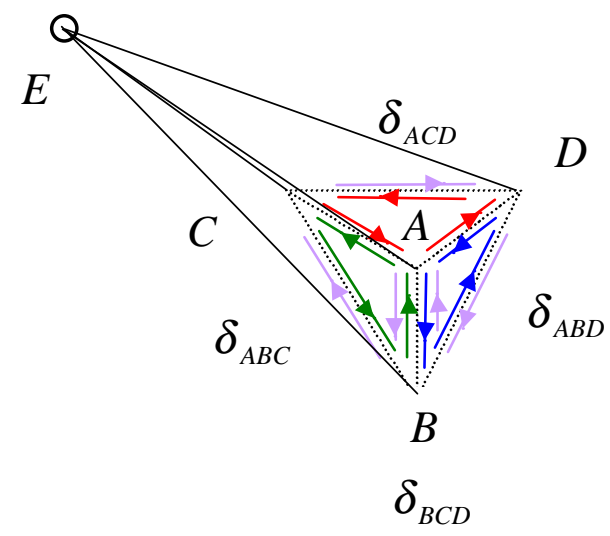

Fig. 6. Similar to Fig. 3., but now in four dimensions. Paths associated with different hinges are shown here in different shades of gray. The product of rotation matrices around a single hinge is simply related to the deficit angle for that hinge. Again, in order to obtain a closed path segment AB has to be transversed more than two times. In constructing a product of rotations, it is sufficient to consider only four of the five vertices; here, vertex $E$ is excluded. In higher dimensional analogues, one has vertices $F, G$, etc., which are also not used.

So, we now consider a path which includes 4 of the 5 simplices which surround the "point in the middle". Specifically, referring to Fig. 6, the path is

$$
A \rightarrow B \rightarrow D \rightarrow A, A \rightarrow D \rightarrow C \rightarrow A, A \rightarrow C \rightarrow B \rightarrow A, A \rightarrow B, B \rightarrow C \rightarrow D \rightarrow B, B \rightarrow A
$$

The same path works for 3-d, which then includes all 4 of the 4 tetrahedra surrounding the point in the middle (see Eq. (3.3)).

\footnotetext{
${ }^{4}$ This three d-simplex result is now easily seen to be true in any number of dimensions for the point in the middle set up.
} 


\section{Product of Rotations in Three Dimensions}

Having described the general geometric setup in the previous section, we now proceed to give an explicit form for the rotation matrices in three dimensions, and derive an exact form of the lattice Bianchi identity by considering a product of rotation matrices along a homotypically trivial path. It turns out to be very convenient to be able to express a rotated vector in terms of the "old" vector and the hinge edge. One notes that the only rotation occurs to the portion of the vector that is perpendicular to the hinge, so that $(\vec{v} \cdot \hat{l}) \hat{l}$ is part of the new vector. One proceeds to form an orthogonal coordinate system with the old vector, $\vec{v}$, and the hinge, $\hat{l}$, using $\hat{l}, \vec{v} \times \hat{l}$ and $\hat{l} \times(\vec{v} \times \hat{l})$. So, since $\vec{v}$ clearly has no component parallel to $\vec{v} \times \hat{l}$, the component of $\mathrm{v}$ which is rotated in the $[\vec{v} \times \hat{l}]-[\hat{l} \times(\vec{v} \times \hat{l})]$ plane is parallel to $\hat{l} \times(\vec{v} \times \hat{l})$. So, one finds

$$
\vec{v}^{\prime}=(\vec{v} \cdot \hat{l}) \hat{l}+\left\{\frac{\vec{v} \cdot[\hat{l} \times(\vec{v} \times \hat{l})]}{|\hat{l} \times(\vec{v} \times \hat{l})|}\right\}\left\{\cos \delta\left[\frac{\hat{l} \times(\vec{v} \times \hat{l})}{|\hat{l} \times(\vec{v} \times \hat{l})|}\right]-\sin \delta\left[\frac{\vec{v} \times \hat{l}}{|\vec{v} \times \hat{l}|}\right]\right\}
$$

which simplifies to

$$
\vec{v}^{\prime}=\left[2 \sin ^{2} \frac{\delta}{2}(\vec{v} \cdot \hat{l})\right] \hat{l}+(\cos \delta) \vec{v}+(\sin \delta) \hat{l} \times \vec{v}
$$

The total rotated vector (after all successive rotations) is most easily found using a recursive application of Eq. (4.2) via standard dot/cross product rules, as well as being sure to set up one's coordinate system where the $\Gamma_{A \rightarrow B}$ in Eq. (3.4) equals 1. Then, one notes that the most general form of a rotation can be written as

$$
R_{t o t} \vec{v}=a \vec{v}+\vec{b} \times \vec{v}+\sum_{i}\left(\vec{c}_{i} \cdot \vec{v}\right) \vec{d}_{i}=\left(\begin{array}{crr}
a+c_{i 1} d_{i 1} & -b_{3}+c_{i 2} d_{i 1} & b_{2}+c_{i 3} d_{i 1} \\
b_{3}+c_{i 1} d_{i 2} & a+c_{i 2} d_{i 2} & -b_{1}+c_{i 3} d_{i 2} \\
-b_{2}+c_{i 1} d_{i 3} & b_{1}+c_{i 2} d_{i 3} & a+c_{i 3} d_{i 3}
\end{array}\right) \vec{v}
$$

where $a, \vec{b}$, and the $\vec{c}_{i}$ are easily found with the expression one had gotten for $\vec{v}_{\text {final }}^{\prime}$ using Eq. (4.2). Here, $\vec{c}_{i}$ runs over all possible vectors in the rotation, which for the sample case we are considering, is just $l_{1}, l_{2}, l_{3}, l_{4}$ and their 6 cross products. The $\vec{d}_{i}$ are then to be viewed as the vector coefficients of the $4+6=10 \vec{c}_{i} \cdot \vec{v}$ 's in Eq. (4.3) (look at the non-matrix expression). The $a$, the $\vec{b}$ and the $\vec{d}_{i}$ are extremely complicated expressions in terms of deficit angles, internal angles, normalized volumes and normalized areas. Here, normalization means dividing by the product of the magnitudes of the defining vectors. Do note that due to the vector nature of the cross product in 3 -d, $a$ does not contain normalized areas.

From the matrix form of Eq. (4.3) one can find a scalar and vector identity. We find these equations by noting

$$
R_{t o t}-I=0
$$


Taking the trace of both sides while using Eq. (4.3) gives the scalar equation

$$
3(a-1)+\sum_{i} \vec{c}_{i} \cdot \vec{d}_{i}=0
$$

while taking the antisymmetric part of Eq. (4.3) gives the vector equation

$$
-\vec{b}-\frac{1}{2} \sum_{i} \vec{c}_{i} \times \vec{d}_{i}=0
$$

and taking the symmetric part of Eq. (4.3) gives a tensor equation which is unnecessary to discuss. So, here, we have found a vector and scalar analogy of the product of rotations for arbitrary deficit angles. These (un-contracted) identities are in terms of deficit angles, internal triangle angles, normalized areas and normalized volumes. The normalized areas, naturally vectors from a geometric point of view, appear explicitly only in the vector identity. Again, it should be noted that Eq. (4.4) is only valid for a product of rotations around hinges which gives no rotation. Permute two of the rotation matrices making up the total rotation, and the new total rotation matrix will not be the unit matrix.

The explicit form of $R_{t o t}$ in Eq. (4.3) can then be used, in combination with Eq. (4.6), to write the 3-dimensional completely contracted Bianchi identities as

$$
-\frac{1}{V(v)} \epsilon_{\alpha \beta \gamma} \epsilon^{\alpha \beta \rho}\left(-\vec{b}-\frac{1}{2} \sum_{i} \vec{c}_{i} \times \vec{d}_{i}\right)_{\rho}=0
$$

which simplifies to

$$
\frac{1}{V(v)}\left(2 \vec{b}+\sum_{i} \vec{c}_{i} \times \vec{d}_{i}\right)_{\gamma}=0
$$

Let us now consider the small deficit angle limit to first order. This simplifies Eq. (4.2) dramatically to

$$
\vec{v}_{\mathrm{small} \delta}^{\prime}=\vec{v}+\delta(\hat{l} \times \vec{v})
$$

which gives, after applying successive rotations,

$$
\begin{aligned}
& a=1 \\
& \vec{b}=\sum_{i} \delta_{i} \hat{l}_{i} \\
& c=0
\end{aligned}
$$

so that Eq. (4.6) becomes

$$
-2 \vec{b}=0
$$

which, following a similar derivation to that of Eq. (4.8), allows the fully contracted Bianchi identities to be written as

$$
\frac{2}{V(v)} \vec{b}_{\gamma}=0
$$


Eq. (4.5) is a trivial $0=0$ to first order. To second order, it becomes

$$
-\vec{b} \cdot \vec{b}=0
$$

where the $\vec{b}$ used is just the $\vec{b}$ in Eq. (4.10). This equation can clearly be seen as a consequence of Eq. (4.11), a first order equation. Eqs. (4.11) and (4.12) (as well as Eq. (8.10), reduced to three dimenions) have been verified to 1st order, and Eq. (4.13) has been verified to 2nd order. But for higher orders, these equations have been shown to be violated in favor of, repectively, Eqs. (4.6), (4.8), and (4.5) (as well as Eq. (8.11), again reduced to three dimensions) which are valid in all cases. Additionally, all these equations are true for arbitrary set-ups, not just our point in the middle set-up.

\section{Product of Rotations in Four Dimensions}

The four-dimensional case is quite similar to the three-dimensional case discussed in the previous section, and no major additional complexities arise. We will give here again an explicit form for the rotation matrices in four dimensions, and derive an exact form of the four-dimensional lattice Bianchi identity by considering a product of rotation matrices along a homotypically trivial path. In the four dimensional case, considering how a vector rotates when it is parallel transported around a hinge, we can, as in the three dimensional case, form again an orthogonal coordinate system using the (old) vector and the hinge, the additional edge in the hinge compensating for going up one dimension. Taking $\hat{l}_{1}$ and $\hat{l}_{2}$ as the two edges which form the hinge, one uses the following four vectors to form the orthogonal coordinate system: $\hat{l}_{1}, \hat{l}_{2}^{\prime}, \vec{v} \times \hat{l}_{1} \times \hat{l}_{2}^{\prime}$ and $\hat{l}_{2}^{\prime} \times \hat{l}_{1} \times\left(\vec{v} \times \hat{l}_{1} \times \hat{l}_{2}^{\prime}\right)$ where

$$
\hat{l}_{2}^{\prime}=\frac{\hat{l}_{2}-\left(\hat{l}_{2} \cdot \hat{l}_{1}\right) \hat{l}_{1}}{\left|\hat{l}_{2}-\left(\hat{l}_{2} \cdot \hat{l}_{1}\right) \hat{l}_{1}\right|}=\frac{\hat{l}_{2}-\left(\hat{l}_{2} \cdot \hat{l}_{1}\right) \hat{l}_{1}}{2 A\left[\hat{l}_{1}, \hat{l}_{2}\right]}
$$

and where $A\left[\hat{l}_{1}, \hat{l}_{2}\right]$ is the area of the triangle, not the parallelogram, formed by $\hat{l}_{1}$ and $\hat{l}_{2}$.

Now, in four dimensions, the hinge we rotate about is a triangle, and the plane in which the rotation occurs is perpendicular to that triangle. Since $\hat{l}_{1}$ and $\hat{l}_{2}^{\prime}$ span the space of the triangle, the components of the rotated vector in those two perpendicular directions will not change. Now, since the only remaining component of the vector is in the $\hat{l}_{2}^{\prime} \times \hat{l}_{1} \times\left(\vec{v} \times \hat{l}_{1} \times \hat{l}_{2}^{\prime}\right)$ direction, v clearly being perpendicular to $\vec{v} \times \hat{l}_{1} \times \hat{l}_{2}$, we can write 
$\vec{v}^{\prime}=\left(\vec{v} \cdot \hat{l}_{1}\right) \hat{l}_{1}+\left(\vec{v} \cdot \hat{l}_{2}^{\prime}\right) \hat{l}_{2}^{\prime}+\left\{\frac{\vec{v} \cdot\left[\hat{l}_{2}^{\prime} \times \hat{l}_{1} \times\left(\vec{v} \times \hat{l}_{1} \times \hat{l}_{2}^{\prime}\right)\right]}{\left|\hat{l}_{2}^{\prime} \times \hat{l}_{1} \times\left(\vec{v} \times \hat{l}_{1} \times \hat{l}_{2}^{\prime}\right)\right|}\right\}\left\{\cos \delta\left[\frac{\hat{l}_{2}^{\prime} \times \hat{l}_{1} \times\left(\vec{v} \times \hat{l}_{1} \times \hat{l}_{2}^{\prime}\right)}{\left|\hat{l}_{2}^{\prime} \times \hat{l}_{1} \times\left(\vec{v} \times \hat{l}_{1} \times \hat{l}_{2}^{\prime}\right)\right|}\right]+\sin \delta\left[\frac{\vec{v} \times \hat{l}_{1} \times \hat{l}_{2}^{\prime}}{\left|\vec{v} \times \hat{l}_{1} \times \hat{l}_{2}^{\prime}\right|}\right]\right\}$

which simplifies to

$$
\vec{v}^{\prime}=2 \sin ^{2} \frac{\delta}{2}\left[\left(\vec{v} \cdot \hat{l}_{1}\right) \hat{l}_{1}+\left(\vec{v} \cdot \hat{l}_{2}^{\prime}\right) \hat{l}_{2}^{\prime}\right]+[\cos \delta] \vec{v}+[\sin \delta] \vec{v} \times \hat{l}_{1} \times \hat{l}_{2}^{\prime}
$$

Now, as in the three dimensional case, we consider null paths as being products of rotations as we parallel transport a vector; only now we are going through 4-simplices as opposed to tetrahedra. Recall that in the three dimensional case, the path could be written in terms of the four tetrahedra it went through, and only depended on the fact that each tetrahedron was directly connected to every other tetrahedron. We can follow the same procedure in the four dimensional case by choosing four of the five 4-simplices and applying Eq. (3.7) (we could interchange 4-simplex E with any of the other 4-simplices in Eq. (3.7)). We have

$$
R_{l_{1}, t o t}-I=\prod_{m=1}^{4} R_{l_{1}, m}-I=0
$$

where the $l_{1}$ is the same as in the previous equations. In general, the $l_{1}$ index can be taken to represent the "hinge base" for the product of rotations, i.e. the edge contained in all hinges involved in the product of rotations; the $l_{2}^{\prime}$ of the previous equations, on the other hand, varies from hinge to hinge. In our set-up, $l_{1}$ is the edge "opposite" the excluded 4 -simplex. (To be sure that one does not have the order of rotations for rotating by $+\delta$ 's reversed, one can either project out along $l_{1}$ or simply try both orders).

Following the 3 -d case, we write the most general product of matrices, and hence of rotation matrices, as

$$
\begin{gathered}
R_{l_{1}, t o t} \vec{v}=a \vec{v}+\sum_{n} \vec{\epsilon}^{-}{ }_{\alpha \beta \gamma} b_{1 n}^{\alpha} b_{2 n}^{\beta} v^{\gamma}+\sum_{i}\left(\vec{c}_{i} \cdot \vec{v}\right) \vec{d}_{i}=a \vec{v}+\sum_{k} \sum_{j_{k}=1}^{\left(j_{k}\right)_{\max }} \vec{\epsilon}^{-}{ }_{\alpha \beta \gamma} b_{1 k}^{\alpha} b_{2 j_{k}}^{\beta} v^{\gamma}+\sum_{i}\left(\vec{c}_{i} \cdot \vec{v}\right) \vec{d}_{i} \\
=\left(\begin{array}{cccc}
a+c_{i}^{1} d_{i}^{1} & \sum_{k j}\left(b_{1 k}, b_{2 j}\right)^{34}+c_{i}^{2} d_{i}^{1} & \sum_{k j}\left(b_{1 k}, b_{2 j}\right)^{42}+c_{i}^{3} d_{i}^{1} & \sum_{k j}\left(b_{1 k}, b_{2 j}\right)^{23}+c_{i}^{4} d_{i}^{1} \\
\sum_{k j}\left(b_{1 k}, b_{2 j}\right)^{43}+c_{i}^{1} d_{i}^{2} & a+c_{i}^{2} d_{i}^{2} & \sum_{k j}\left(b_{1 k}, b_{2 j}\right)^{14}+c_{i}^{3} d_{i}^{2} & \sum_{k j}\left(b_{1 k}, b_{2 j}\right)^{31}+c_{i}^{4} d_{i}^{2} \\
\sum_{k j}\left(b_{1 k}, b_{2 j}\right)^{24}+c_{i}^{1} d_{i}^{3} & \sum_{k j}\left(b_{1 k}, b_{2 j}\right)^{41}+c_{i}^{2} d_{i}^{3} & a+c_{i}^{3} d_{i}^{3} & \sum_{k j}\left(b_{1 k}, b_{2 j}\right)^{12}+c_{i}^{4} d_{i}^{3} \\
\sum_{k j}\left(b_{1 k}, b_{2 j}\right)^{32}+c_{i}^{1} d_{i}^{4} & \sum_{k j}\left(b_{1 k}, b_{2 j}\right)^{13}+c_{i}^{2} d_{i}^{4} & \sum_{k j}\left(b_{1 k}, b_{2 j}\right)^{21}+c_{i}^{3} d_{i}^{4} & a+c_{i}^{4} d_{i}^{4}
\end{array}\right) \vec{v}(5)
\end{gathered}
$$

where

$$
\left(b_{1 k}, b_{2 j}\right)^{\alpha \beta} \equiv b_{1 k}^{\alpha} b_{2 j}^{\beta}-b_{1 k}^{\beta} b_{2 j}^{\alpha}
$$


The main difference between Eq. (5.5) and Eq. (4.3) is that in Eq. (4.3) there was only one $\vec{b}$, whereas here, we have a set of pairs of $\vec{b}$ 's, i.e. $\left\{\left(\vec{b}_{1 n}, \vec{b}_{2 n}\right)\right\}$. So, for these new $\vec{b}$ 's, the first lower index indicates whether it comes 1 st or 2 nd in the cross product with $\vec{v}$, the second lower index indicates what term in the sum it belongs to, and the upper index indicates which component of it is being taken. In our point in the middle set up, the $\vec{c}_{i}$ 's consist of 5 edges and their 10 triple cross products; there are 15 corresponding $\vec{d}_{i}$ 's. The $\sum_{n}$ form has a nicer appearance, but the $\sum_{k j}$ form is much better when doing computations. The $\mathrm{k}$ subscript in $j_{k}$ has been omitted in the matrix form given.

Using Eq. (5.4) and taking the trace and the antisymmetric part of the equation give, respectively, ${ }^{5}$

$$
4(a-1)+\sum_{i} \vec{c}_{i} \cdot \vec{d}_{i}=0
$$

and

$$
B-\frac{1}{2} C=0
$$

where $\mathrm{B}$ and $\mathrm{C}$ have matrix elements ${ }^{6}$

$$
B^{\alpha \beta} \equiv \sum_{j k} \epsilon_{\gamma \delta}^{\alpha \beta} b_{1 j}^{\gamma} b_{2 k}^{\delta}
$$

and

$$
C^{\alpha \beta} \equiv \sum_{i}\left(c_{i}^{\alpha} d_{i}^{\beta}-d_{i}^{\alpha} c_{i}^{\beta}\right)
$$

These (un-contracted) identities are in terms of deficit angles, internal triangle angles, normalized areas and normalized four-volumes. The normalized areas, naturally 2-index tensors, appear explicitly only in the tensor identity, Eq. (5.8).

Using the explicit form of $R_{l_{1}, t o t}$ in Eq. (5.5) and Eq. (5.8) the completely contracted Bianchi identities can then be written as

$$
-\frac{1}{2} \frac{1}{{ }^{4} V(v)} \sum_{l_{1} \supset v} \epsilon_{\alpha \beta \gamma \lambda}(2 B-C)^{\alpha \beta} l_{1}^{\lambda}=0
$$

To first order in deficit angles, Eqs. (5.7) and (5.8) reduce to, respectively, $0=0$ and

$$
B^{\alpha \beta}=\sum_{i} \delta_{i} \epsilon_{\mu \nu}^{\alpha \beta} l_{1}^{\mu} l_{2 i}^{\prime \nu}=0
$$

\footnotetext{
${ }^{5}$ In $d$ dimensions, $4(1-a) \rightarrow d(1-a)$.

${ }^{6}$ In $d$ dimensions, there are $d-2 b$ 's contracted with the Levi-Civita tensor.
} 
The above equation can be rewritten, using Eq. (2.4), to give

$$
\sum_{i} \delta_{i} U_{i}^{\alpha \beta}=0
$$

where we have substituted in for $l_{i}^{\prime}$ using Eq. (5.1). Following a similar derivation to that of Eq. (5.11), the above equation allows the fully contracted Bianchi identities to be written for small angles as

$$
-\frac{1}{{ }^{4} V(v)} \sum_{l_{1} \supset v} \epsilon_{\alpha \beta \gamma \lambda}\left(\sum_{i} \delta_{i} U_{i}^{\alpha \beta}\right) l_{1}^{\lambda}=0
$$

Eqs. (5.13), (5.14) (as well as the Eq. (8.10), reduced to four dimensions) have been verified to first order. For higher order, these equations have been shown to be violated in favor of, respectively, Eqs. (5.8) and (5.11) (as well as Eq. (8.11), again reduced to four dimensions). Also, Eq. (5.7), a trivial $0=0$ to first order, has been shown to be true generally. ${ }^{7}$

\section{Discrete Riemann Tensor and its Dual}

In the previous two sections an explicit, exact form of the lattice Bianchi identity was derived by considering a product of rotation matrices (in three and four dimensions respectively) along a homotypically trivial path. Curvature enters the above lattice Bianchi identities through the deficit angle, but one interesting question left partially open is the relationship between the exact lattice Bianchi identities (which involve products of rotation matrices) and the continuum Bianchi identities (which involve derivatives of the Riemann tensor). In this and the following section an expression of the Riemann tensor and its dual will be derived, which will eventually be used to show that in four dimensions (Section 9), if one proceeds from the continuum Bianchi identities (in integrated form) and inserts the expression for the discrete Riemann tensor discussed below one

\footnotetext{
${ }^{7}$ We note here that these results apply to the Lorentz case as well. One sets $c t=-i x_{4}$, and finds the $\vec{x}, t$ rotations equivalent to the $\vec{x}, x_{4}$ rotations. Since all the Bianchi products of rotations, when written out in terms such as the $\Gamma_{A \rightarrow B}$ gauge transformation of Section 3, end up canceling to the unit matrix via products such as $\Gamma_{A \rightarrow B} \Gamma_{A \rightarrow B}^{-1}$, and as each such Euclidean gauge transformation has an equivalent Lorentz transformation, the Regge Calculus Bianchi identities hold in the Lorentz case as well. In finding these equivalent transformations, it is important to note that any rotation plane will have at least one spatial axis. If the other (perpendicular) axis is also spatial, one has the standard rotation by the computed deficit angle. If the other axis is time-like, one first computes the deficit angle as in the Euclidean case, multiplies it by $i$ to get the appropriate deficit angle to use in the sines and cosines, and ends up using hyperbolic sines and cosines. For this case, it is important not to subtract out any multiple $2 \pi$ from the original Euclidean deficit angle before it is multiplied by $i$. If the other axis is null, there is no rotation, no matter what the deficit angle would be in the Euclidean case, as a null vector and a spatial vector cannot rotate into each other. These three cases are, respectively, equivalent to $A_{\Gamma}^{\mu \nu} A_{\mu \nu}^{\Gamma}>0, A_{\Gamma}^{\mu \nu} A_{\mu \nu}^{\Gamma}<0$ and $A_{\Gamma}^{\mu \nu} A_{\mu \nu}^{\Gamma}=0$; alternatively, they are also equivalent to, respectively, $A_{h}^{\gamma \delta_{1} \ldots \delta_{d-3}} A_{\gamma \delta_{1} \ldots \delta_{d-3}}^{h}<0, A_{h}^{\gamma \delta_{1} \ldots \delta_{d-3}} A_{\gamma \delta_{1} \ldots \delta_{d-3}}^{h}>0$ and $A_{h}^{\gamma \delta_{1} \ldots \delta_{d-3}} A_{\gamma \delta_{1} \ldots \delta_{d-3}}^{h}=0$. Lastly, it is important to note that, once one fixes a going around coordinate system, one needs Euler angles to write a space-time rotation.
} 
obtains, after promoting infinitesimal rotations to finite rotations, the same exact lattice Bianchi identity discussed previously in Section 4 (in Section 10 the same set of result is presented in three dimension).

Having obtained the necessary ingredients to describe arbitrary lattice rotations of vectors, we proceed next to derive a general form for the lattice Riemann curvature tensor. An explicit form for the Riemmann tensor in term of rotations will be quite useful here, since it will allow us to establish a relationship (known to exist in the continuum) between the Regge field equations and the lattice Bianchi identities derived in the previous sections in three and four dimensions. Furthermore it will allow us to check the overall consistency of our results, since later in the paper we will re-derive the lattice Bianchi identities by starting from their continuum expression in terms of the Riemann tensor, and by promoting infinitesimal rotations to finite rotations will be able to show that the resulting lattice Bianchi identities are in fact identical (in $d=3$ and $d=4$ ) to the expressions previously derived in the preceding sections.

Consider moving a vector $V$ once around a Voronoi loop, i.e. a loop formed by Voronoi edges surrounding a hinge. The change in $V$, denoted here by $\delta V$, is then given by

$$
\delta V^{\alpha}=(\mathbf{R}-\mathbf{1})^{\alpha}{ }_{\beta} V^{\beta}
$$

where $\mathbf{R}$ is the is the rotation matrix associated with the hinge. Now, $\delta V$ in the continuum is given by

$$
\delta V^{\alpha}=\frac{1}{2} R_{\beta \mu \nu}^{\alpha} A_{\Gamma}^{\mu \nu} V^{\beta}
$$

where $A_{\Gamma}^{\mu \nu}$ is the antisymmetric bivector representing the loop area. So we make the tentative identification

$$
\frac{1}{2} R_{\beta \mu \nu}^{\alpha} A_{\Gamma}^{\mu \nu}=(\mathbf{R}-\mathbf{1})_{\beta}^{\alpha}
$$

Now, because of the sums on $\mu$ and $\nu$, it is not immediately clear how one can divide by $A_{\Gamma}^{\mu \nu}$ to solve for the Riemann curvature tensor. Let us, then, simply take a frame where there are only two components of $A_{\Gamma}^{\mu \nu}$, namely $A_{\Gamma}^{12}$ and $A_{\Gamma}^{21}$. In that frame, using the antisymmetry of Riemann in $\mu$ and $\nu$, one can divide, and one finds

$$
R_{\beta 12}^{\alpha}=\frac{(\mathbf{R}-\mathbf{1})^{\alpha}{ }_{\beta}}{A_{\Gamma}^{12}}
$$


Multiplying by unity in the form of $A_{12}^{\Gamma}$ (the ${ }_{12}$ component of the index-lowered version of $A_{\Gamma}^{12}$ ) over itself, one finds

$$
R_{\beta 12}^{\alpha}=\frac{(\mathbf{R}-\mathbf{1})^{\alpha}{ }_{\beta} A_{12}^{\Gamma}}{A_{\Gamma}^{2}}
$$

where the area of the loop is an invariant satisfying $A_{\Gamma}^{2}=A_{\lambda \kappa}^{\Gamma} A_{\Gamma}^{\lambda \kappa} / 2$. Of course, all values of the Riemann curvature tensor with $\alpha$ or $\beta$ neither 1 or 2 are zero in this coordinate system.

Now, the above equation can be rewritten in this coordinate system as

$$
R_{\beta \mu \nu}^{\alpha}=\frac{(\mathbf{R}-\mathbf{1})^{\alpha}{ }_{\beta} A_{\mu \nu}^{\Gamma}}{A_{\Gamma}^{2}}
$$

However, this is now a tensor equation, and hence valid in all coordinate systems. Still, there is a basic problem with this, and that is that $(\mathbf{R}-\mathbf{1})_{\sigma \beta}$ is only antisymmetric to first order (for example, $(\mathbf{R}-\mathbf{1})_{11}=0$ only to first order), so that this identification of the right hand side of Eq. (6.6) with the Riemann curvature tensor and all of its symmetries can only be valid to first order. So, let us write Eq. (6.6) to first order in the deficit angle $\delta$ :

$$
R_{\beta \mu \nu}^{\alpha}=\frac{\delta}{A_{\Gamma}} U_{\beta}^{\alpha} \frac{A_{\mu \nu}^{\Gamma}}{A_{\Gamma}}
$$

where, in our coordinate system, which we now take to be orthonormal, ${ }^{8}$

$$
U_{\alpha \beta}=\left(\begin{array}{rrrr}
0 & -1 & 0 & 0 \\
1 & 0 & 0 & 0 \\
0 & 0 & 0 & 0 \\
0 & 0 & 0 & 0
\end{array}\right)
$$

The general form of $U_{\alpha \beta}$, in an arbitrary coordinate system, is

$$
U_{\alpha \beta}=\epsilon_{\alpha \beta \mu \nu} l_{1}^{\mu} l_{2}^{\nu} / 2 A_{h}
$$

where $l_{1}$ and $l_{2}$ are the two hinge vectors and $A_{h}=\sqrt{A_{\lambda \kappa}^{h} A_{h}^{\lambda \kappa} / 2}$ with $A_{h}$ the area of the hinge and $A_{h}^{\lambda \kappa}$ the associated bivector. ${ }^{9}$ Note that $\delta$ is invariant because it is formed from angles which are arc cosines of ratios of invariant dot products to invariant lengths, and furthermore that $\epsilon_{\alpha \beta \mu \nu}=$ $\sqrt{g}[1234]$ with $[1234]=+1$. So Eq. (6.9) is a tensor, and hence so is Eq. (6.7). Since the expression in Eq. (6.7), after lowering the first index, also has the appropriate antisymmetry in the first two indices, we can see it as a Riemann tensor candidate.

\footnotetext{
${ }^{8} \mathrm{~A}$ minus sign appears above in the Lorentz case of a $\mathrm{x}$-t loop.

${ }^{9}$ In the Lorentzian case of a space-time hinge, $A_{h}=\sqrt{-A_{\lambda \kappa}^{h} A_{h}^{\lambda \kappa} / 2}$.
} 
Now, by going back to our special coordinate system, it can be easily verified that the dual of the normalized loop area is the normalized hinge area, i.e.

$$
\frac{A_{\alpha \beta}^{\Gamma}}{A_{\Gamma}}=\frac{\epsilon_{\alpha \beta \gamma \delta}}{2} \frac{A_{h}^{\gamma \delta}}{A_{h}}
$$

This allows us to rewrite Eq. (6.7) simply as

$$
R_{\beta \mu \nu}^{\alpha}=\frac{\delta}{A_{\Gamma}} U_{\beta}^{\alpha} U_{\mu \nu}
$$

This expression has been discussed by the authors of Ref.[7, 10], and is known to possess all requisite symmetry properties of the Riemann tensor. However, as these authors note, it implies that the square of the Ricci scalar, the square of the Ricci tensor and the square of the Riemann tensor are all directly proportional, with constants of proportionality independent of the edge lengths. We now define

$$
R_{\beta \mu \nu}^{\alpha} \equiv R_{\beta \mu \nu}^{\alpha}(b, v) \equiv \sum_{\substack{h \subset b \\ h \supset v}} R_{\beta \mu \nu}^{\alpha}(h)
$$

for a formulation of Regge Calculus which breaks up space-time into d-boxes. Here, one chooses one particular vertex $v$ to be the origin for the $g_{\mu \nu}$ of d-box $b$. Then the metric can, for example, be defined via dot products of the box's non-diagonal edges containing $v$, or, equivalently, can be defined via edges in the box containing $v$ which are in one of the box's d-simplices. Other vertices $v$ for other d-boxes are chosen to be in the same relative location within the d-boxes, so that each hinge curvature term $R_{\beta \mu \nu}^{\alpha}(h)$ is included once.

Two other definitions of the Riemann tensor are possible in principle, but as we will be show below, fail to pass a crucial test. Noting that each rotation plane has four hinges for a d-box, one could have alternately defined

$$
R_{\beta \mu \nu}^{\alpha} \equiv \frac{1}{4} \sum_{h \subset s} R_{\beta \mu \nu}^{\alpha}(h)
$$

This could be useful in a situation with large curvatures where the increased computation due to averaging and, as we will see, more complex Einstein tensors $G_{\gamma} \gamma^{\prime}$ 's, is more than canceled out by being able to use fewer d-boxes due to the relaxing of the origin vertex location constraint.

Alternatively, if one uses only d-simplices and does not require them to form d-boxes, then one can define

$$
R_{\beta \mu \nu}^{\alpha} \equiv R_{\beta \mu \nu}^{\alpha}(s, v) \equiv \sum_{h \subset s} \frac{1}{N_{s_{h}}} R_{\beta \mu \nu}^{\alpha}(h)
$$

where $N_{s_{h}}$ is the number of d-simplices containing hinge $h$.

Now, one objection to these definitions is that the number of independent curvature components used per d-volume, for these three cases respectively (Eqs. (6.12), (6.13) and (6.14)), is $d(d-1) / 2$, 
$2 d(d-1)$ and $d(d+1) / 2$, none of which is the expected $d^{2}\left(d^{2}-1\right) / 12$, the number of components of Riemann in the continuum. However, let us now calculate the number of components of Riemann about a vertex in the d-box case where consistent labeling of the origins are possible, i.e. where $2^{d}$ d-boxes surround each vertex. The number of m-boxes surrounding a given vertex is $2^{m}(\stackrel{d}{m})$ where we choose $m$ of the $d$ axis directions, and note that there are two choices per direction. Setting $m=d-2$ gives

$$
\begin{aligned}
N_{h, v}=2^{d-3} d(d-1) & =d^{2}\left(d^{2}-1\right) / 12 \quad d=2,3 \\
& >d^{2}\left(d^{2}-1\right) / 12 \quad d \geq 4
\end{aligned}
$$

So, if one sets up a going around coordinate system at a vertex, and defines the curvature via

$$
R_{\beta \gamma \delta}^{\alpha} \equiv \sum_{h \supset v} R_{\beta \gamma \delta}^{\alpha}(h)
$$

the symmetries of the form of the hinge curvatures keep the number of independent components of the curvature equal to $d^{2}\left(d^{2}-1\right) / 12$ in any dimension. This equation, then, having along with the natural form for the Riemann curvature tensor, all the symmetry properties of the continuum Riemann curvature tensor as well as the correct number of independent components, is the Regge analog of the Riemann curvature tensor. ${ }^{10}$

We are now in a position to calculate the dual Riemann tensor via

$$
\tilde{R}^{\alpha \beta \gamma \delta} \equiv \frac{1}{2} \epsilon^{\mu \nu \gamma \delta} R_{\mu \nu}^{\alpha \beta}=\frac{1}{2} \delta \epsilon^{\mu \nu \gamma \delta} U^{\alpha \beta} A_{\mu \nu}^{\Gamma} / A_{\Gamma}^{2}
$$

where the loop now used is one of the aforementioned inner-d-box loops. ${ }^{11}$ We can interpret the above result in the following way. Here $U_{\beta}^{\alpha}$ corresponds to the "effective" $U_{\beta}^{\alpha}$ of the loop, and $\delta$ is the "effective" deficit angle of the loop. The "effective" Riemann curvature tensor is the sum of all the "old" Riemann curvature tensors corresponding to the areas that the $2^{d}-1=15$ edges form, so that the effective $\delta$ and $U^{\alpha \beta}$ are determined for each standard square loop (which can only encircle areas formed by the 15 edges). Note that, since our "old" Riemann curvature tensors had

\footnotetext{
${ }^{10}$ Were we to use d-simplices which need not form d-boxes, then the consistent choice of origin vertices may be either impossible or very difficult. One might also make this choice for a d-box situation with high curvatures where the increased computation due to averaging is more than canceled out by being able to use fewer d-boxes. But this is more complicated and our results for $G_{\gamma}{ }^{\prime}$ will also show a distinct preference for the first option. Still, in cases where curvature is sufficiently high and computing power will not allow for a sufficient breakdown of space-time into d-boxes such that a consistent choice of origin vertices is possible, this definition may be useful. Indeed, for this reason, were one to break down space-time into d-simplices without forming d-boxes, this definition would likely be necessary. Still, in such a case where either a quick estimate is needed or where computing power is insufficient to use the first definition, this definition would then be useful.

${ }^{11}$ In the Lorentzian case, there is a minus sign in this definition of $\tilde{R}^{\alpha \beta \gamma \delta}$.
} 
all the symmetry properties of the continuum Riemann curvature tensor, and because our "new" (i.e. actual) Riemann curvature tensor is the sum of these old tensors, it too has all the symmetry properties of the continuum Riemann curvature tensor. And, further, it has the advantage of allowing us to consider, in the case of small deficit angles, only perpendicular loops (more precisely, perpendicular loop areas), as we do in the continuum. Now, using Eq. (6.10), Eq. (6.18) can be written as

$$
\tilde{R}^{\alpha \beta \gamma \delta}=\frac{\delta}{A_{\Gamma}} U^{\alpha \beta} \frac{A_{h}^{\gamma \delta}}{A_{h}}
$$

which, incidentally, holds in any number of dimensions simply by adding $d-4$ extra indices after the $\delta$ indices. As the action will involve an integration, it will now be helpful to find out the total four-volume enclosed by the hinge and its loop in terms of the product of the loop area and the hinge area.

In the 4 -d case the denominator, $A_{\Gamma} A_{h}$, is six times the four-volume enclosed by the hinge and the path, here denoted by ${ }^{4} V(h, \Gamma)$, this latter four-volume being formed via the Voronoi vertices surrounding the hinge and the hinge vertices. To see this fact, note that, in the 3 -d case, as one approaches a vertex, the area perpendicular to the hinge is similar to the loop area, but gets smaller proportionally to the square of the perpendicular distance from the point, labeled $p$, which marks the intersection of the loop area and the hinge. (In particular, all lengths defining the area are proportional to this distance, and hence areas, as functions of products of pairs of lengths, must be proportional to the square of this distance.) By letting $l_{1 a}$ be the distance from one of $l_{1}$ 's vertices to $p$, and letting $l_{1 b}$ be the distance from $l_{1}$ 's other vertex to $p$, one finds the total 3 -d volume formed by the loop and $l_{1}$ to be

$$
{ }^{3} V=\int_{0}^{l_{1 a}}\left(A_{\Gamma}\right)\left(s / l_{1 a}\right)^{2} d s+\int_{0}^{l_{1 b}}\left(A_{\Gamma}\right)\left(s / l_{1 b}\right)^{2} d s=A_{\Gamma} l_{1} / 3
$$

In 4-d, the total four-volume formed by the loop and the hinge is obtained by noting that the three volume shrinks proportionally to the cube of the perpendicular distance from the three volume to the remaining vertex, so that, letting $l_{2}^{\delta}$ be the component of the remaining hinge edge which is perpendicular to the tetrahedron,

$$
{ }^{4} V=\int_{0}^{l_{2}^{\delta}}\left(A_{\Gamma} l_{1} / 3\right)\left(s / l_{2}^{\delta}\right)^{3} d s=\epsilon_{\mu \nu \gamma \delta} A_{\Gamma}^{\mu \nu} l_{1}^{\gamma} l_{2}^{\delta} / 24=\epsilon_{\mu \nu \gamma \delta} A_{\Gamma}^{\mu \nu} A_{h}^{\gamma \delta} / 24=A_{\Gamma} A_{h} / 6
$$

where the next to last term is found by taking our special coordinate system, with two axes along $A_{\Gamma}$ and two axes along $A_{h}$. In d-dimensions, one has ${ }^{d} V=\epsilon_{\mu \nu \gamma \delta \ldots} A_{\Gamma}^{\mu \nu} V_{h}^{\gamma \delta \ldots} / d !=2 A_{\Gamma} V_{h} / d(d-1)$. 
Incidentally, the only constraint on the loop here is that it be perpendicular to the hinge. One has therefore

$$
\tilde{R}^{\alpha \beta \gamma \delta}=\frac{1}{6} \frac{\delta}{{ }^{4} V_{h}} U^{\alpha \beta} A_{h}^{\gamma \delta}
$$

Using Eq. (6.11), we find

$$
R=R_{\alpha \beta}^{\alpha \beta}=\frac{\delta}{A_{\Gamma}} \frac{\epsilon_{\alpha \beta \gamma_{1} \ldots \gamma_{d-2}} A_{h}^{\gamma_{1} \ldots \gamma_{d-2}} \epsilon^{\alpha \beta \gamma_{1}^{\prime} \ldots \gamma_{d-2}^{\prime}} A_{\gamma_{1}^{\prime} \ldots \gamma_{d-2}^{\prime}}^{\prime}}{\left[(d-2) ! A_{h}\right]^{2}}=2 \frac{\delta}{A_{\Gamma}}
$$

so that the Einstein-Hilbert action becomes

$$
I=-\int_{X} R \sqrt{g} d^{d} x=-\sum_{h} \int_{h} \frac{2 \delta}{A_{\Gamma}} \sqrt{g} d^{d} x
$$

where $X$ is all space and $\int_{h}$ is defined as the integral over ${ }^{d} V\left(h, \Gamma_{h}\right)$, the d-volume formed by the hinge and the loop area. As the integrand is constant over the hinge, we do the integral by simply calculating this aforementioned d-volume. One can now can write the action as

$$
I=-\frac{4}{d(d-1)} \sum_{h} \delta_{h} V_{h}
$$

where $V_{h} / d(d-1)$ is the (d-2)-volume of the hinge, reducing to an area $A_{h}$ in 4 -d. (The sign in the above equation becomes positive in the Lorentz case.)

\section{Action Variation and Einstein Tensor}

This section will be devoted to discussing the relationship between the expression for the dual of the Riemann tensor, as given in the previos section, and the Regge field equations. We will show that the above construction is indeed consistent, by deriving from it the Regge field equations. While this does not constitute a general proof of correctness of the proposed expression, it does provide one rather significant test.

Next one would like to vary the action with respect to $g_{\mu \nu}$. In the continuum each $g_{\mu \nu}$ is an independent variable upon which the geometry is based, which is why one varies the action with respect to them. Since, in Regge Calculus, the geometry is completely based on edge lengths, only edge lengths can be used in computing $g_{\mu \nu}$. In particular, any $g_{\mu \nu}$ for the loop plane is not a true $g_{\mu \nu}$, since these quantities can be defined independent of edge lengths. Furthermore, one can see that if one did vary the above action with respect to such a quantity, one would get zero because 
the hinge areas are independent of any quantity which is independent of the edge lengths. So, one is led to consider the metric $g_{\mu \nu}$ as being defined as a function of hinge edge lengths. Here, we take one $(d-2) \times(d-2) g_{\mu \nu}$ matrix per hinge. Using the invariance of $\delta$ in any dimension[1], ${ }^{d} V_{h}$ as the Voronoi d-volume surrounding the hinge and $V_{h}=\int_{h} \sqrt{|g|} d^{d-2} x /(d-2)$ !, we now compute, for an individual hinge,

$$
\begin{aligned}
\mathcal{G}_{h \gamma}{ }^{\gamma^{\prime}} & \equiv \int_{{ } V_{h}} G_{h \gamma^{\gamma^{\prime}}} \sqrt{|g|} d^{d} x=-\frac{4}{d(d-1)} g_{\gamma \rho} \delta_{h} \frac{\partial V_{h}}{\partial g_{\rho \gamma^{\prime}}} \\
& =-\int_{h} \frac{4}{d !} \delta_{h} \frac{g_{\gamma \rho}}{2 \sqrt{|g|}}\left[\sigma_{1} \ldots \sigma_{d-2}\right] g_{\sigma_{1} 1 \ldots} g_{\sigma_{\gamma^{\prime}-1} \gamma^{\prime}-1} \delta_{\sigma_{\gamma^{\prime}}} g_{\sigma_{\gamma^{\prime}+1} \gamma^{\prime}+1} \ldots g_{\sigma_{d-2} d-2} d^{d-2} x \\
& =-\frac{2}{d(d-1)} \delta_{\gamma}^{\gamma^{\prime}} \delta_{h} V_{h}
\end{aligned}
$$

To validate this result, ${ }^{12}$ which confirms the correctness of using $(d-2) \times(d-2)$ hinge metrics, we compute the above quantity in a different manner via ${ }^{13}$

$$
\begin{aligned}
\mathcal{G}_{h \gamma}{ }^{\gamma^{\prime}} & =\int_{{ }^{V_{h}}} G_{h \gamma} \gamma^{\prime} \sqrt{|g|} d^{d} x=-\frac{1}{2(d-3) !} \int_{{ }^{V_{h}}} \epsilon_{\alpha \beta \gamma^{\prime} \delta_{1} \ldots \delta_{d-3}} \tilde{R}_{h}^{\alpha \beta \gamma \delta_{1} \ldots \delta_{d-3}} \sqrt{|g|} d^{d} x \\
& =-\frac{1}{2(d-3) !} \frac{{ }^{d} V_{h}}{A_{\Gamma} V_{h}} \epsilon_{\alpha \beta \gamma^{\prime} \delta_{1} \ldots \delta_{d-3}} \delta_{h} U^{\alpha \beta} V_{h}^{\gamma \delta_{1} \ldots \delta_{d-3}}=-\frac{2}{d(d-1)} \delta_{\gamma}^{\gamma^{\prime}} \delta_{h} V_{h}
\end{aligned}
$$

Now, noting that this final result is independent of the metric, and noting that the $\delta_{\gamma} \gamma^{\prime}$ indicates that the components of $\mathcal{G}_{\gamma} \gamma^{\prime}$ are most naturally taken along edges, we find

$$
\mathcal{G}_{l} l^{\prime}=-\frac{2}{d(d-1)} \sum_{\substack{h \supset l \\ h \subset \mathrm{d}-\text { box }}} \delta_{h} V_{h} \delta_{l}^{l^{\prime}}
$$

In a given d-box of our global coordinate system, where these l's are the box's (non-diagonal) axis $l$, the integrated Einstein tensor is defined via hinges within that d-box, so that there is no global over-counting of each contribution to $\mathcal{G}_{l}{ }^{l}$. We now easily see that, using our d-box's $g_{\mu \nu}$ defined by the axes' edges,

$$
\mathcal{G}_{l l^{\prime}}=-\frac{2}{d(d-1)} \sum_{\substack{h \supset l \\ h \subset \mathrm{d}-\mathrm{box}}} \delta_{h} V_{h} g_{l l^{\prime}} \quad \text { and } \quad \mathcal{G}^{l l^{\prime}}=-\frac{2}{d(d-1)} \sum_{\substack{h \supset l \\ h \subset \mathrm{d}-\mathrm{box}}} \delta_{h} V_{h} \delta^{l l^{\prime}}
$$

The cosmological constant term is derived in each d-box from the d-box's $g_{\mu \nu}$ via the variation of $2 \int \lambda \sqrt{g} d^{d} x$ exactly as it is in the continuum to get

$$
\lambda g^{l l^{\prime}} \text { and hence } \lambda \delta_{l}^{\lambda^{\prime}} \text { and } \lambda g g_{l l^{\prime}}
$$

\footnotetext{
${ }^{12}$ The sign is opposite for a space-time loop, because Eq. (6.25) changes sign for the reason given in the footnote for Eq. (6.7). Also, letting $\sqrt{g} \rightarrow \sqrt{-g}$ for space-time hinges has no effect on the sign.

${ }^{13}$ Once again, the sign of the result changes for a space-time loop for the reason given in the footnote to Eq. (6.7). For any Lorentzian hinge, the minus sign referenced in the footnote to Eq.(6.18) is canceled by the minus sign in the inverse to Eq. (6.10).
} 
This is the most natural result for the left hand side of Einstein's equations for Regge Calculus because of its metric independence. A computation of $\mathcal{G}^{\mu \nu}$ or $\mathcal{G}_{\mu \nu}$ for edges would have been counter-intuitive because one would be using a different metric for each hinge in the sum. Also, using $G_{h \gamma}{ }^{\gamma^{\prime}}$ would have explicitly involved the areas of the hinges' loops, which are not uniquely defined. Now, the standard result for the field equations in four dimensions

$$
\frac{l}{2} \sum_{h \supset l} \delta_{h} \cot \theta_{o p}
$$

is derived by varying the integrated action with respect to the edge lengths, and hence is most similar to $\mathcal{G}^{\mu \nu}$, which is found by varying with respect to $g_{\mu \nu}$, which is a function of squares of edge lengths. Hence this standard result is not metric independent. However, as long as one has $T^{\mu \nu}=0$, there is no problem with it as Regge Calculus then only involves edge lengths. However, if one wishes to include $T^{\mu \nu}$, and if this $T^{\mu \nu}$ is not derivable from an action, then one must use Eq. (7.3). Also, Eq. (7.3) seems more natural, and, except for having a more limited sum, has the same form as the action. Lastly, we note that the individual terms in Eqs.(7.3) and (7.6) can be related via the transformations $g_{11}=l_{1}^{2}, g_{22}=l_{2}^{2}$ and $g_{12}=\left(l_{1}^{2}+l_{2}^{2}-L^{2}\right) / 2$ so that the variation of the integral of the action can be transformed, for any one fixed term, between the two sets of variables via a Jacobian.

Ideally, one wants a one-to-one mapping between the (independent) edge length variables and the independent $g_{\mu \nu}$ variables. This is easily achieved by choosing as the independent $g_{\mu \nu}$ 's the $g_{\mu \mu}$ 's, i.e. the metric components equal to the square of a given edge length. Of course, we can always switch global metric variables by replacing some $g_{\mu \mu}$ 's with an equal number of $g_{\mu \nu}$ 's (while being sure to maintain the relative independence of the new set of metric components). We do this now, choosing $g_{11}=l_{1}^{2}$ for all $(d-2) \times(d-2)$ metrics for the hinges bordering $l_{1}$; we let all such metrics be based at one of $l_{1}$ 's vertices. All other lattice $g_{\mu \nu}$ 's are then chosen such that the set of all $g_{\mu \nu}$ 's are independent. A simple way to make such a choice is to choose the metric components equal to the edge length squared of each edge not in a simplex bordering $l_{1}$. Now, replacing $l_{1}$ with $L$ and varying with respect to $g_{11}=L^{2}$ gives, for 4 -d

$$
-\frac{1}{12} \sum_{h \supset L} \delta_{h} \cot \theta_{o p}
$$

which is easily related to the standard result by multiplying by $-6 L$.

As an exercise we show next the equivalence of our Einstein tensor to the variation of the action with respect to the edge lengths in the case of four dimensions. For a hinge with edges $l_{1}$ and $l_{2}$, taking $g_{11}=l_{1}^{2}, g_{22}=l_{2}^{2}$ and $g_{12}=l_{1} \cdot l_{2}=\left(l_{1}^{2}+l_{2}^{2}-L^{2}\right) / 2$ where $L$ is the third edge of the triangle 
formed by $l_{1}$ and $l_{2}$, we have

$$
A=\frac{1}{2} \sqrt{g_{11} g_{22}-g_{12} g_{21}}
$$

where, for variation purposes, we take $g_{12}$ and $g_{21}$ to be independent. This implies

$$
\begin{array}{cc}
\frac{\partial A}{\partial g_{11}}=\frac{g_{22}}{8 A} & \frac{\partial A}{\partial g_{22}}=\frac{g_{11}}{8 A} \\
\frac{\partial A}{\partial g_{12}}=-\frac{g_{21}}{8 A} & \frac{\partial A}{\partial g_{21}}=-\frac{g_{12}}{8 A}
\end{array}
$$

and therefore

$$
\frac{\partial A}{\partial L}=\frac{\partial A}{\partial g_{\mu \nu}} \frac{\partial g_{\mu \nu}}{\partial L}=\frac{g_{12}}{4 A}=\frac{L}{2} \cot \theta_{o p}
$$

where we have used $g_{12}=l_{1} l_{2} \cos \theta_{o p}, A=l_{1} l_{2} \sin \theta_{o p} / 2, g_{12}=\left(l_{1}^{2}+l_{2}^{2}-L^{2}\right) / 2, g_{11}=l_{1}^{2}$ and $g_{22}=l_{2}^{2}$. This is consistent with the standard Regge Calculus result for the field side of the Einstein field equations. Also, similar results apply for either of the other two edges if we change the metric so that its diagonal components do not have that edge. If we do not change the metric, we find, for e.g., for $l_{1}$,

$$
\frac{\partial A}{\partial l_{1}}=\frac{1}{4}\left(l_{2} \csc \theta_{o p}-l_{1} \cot \theta_{o p}\right)
$$

where $\theta_{o p}$ is still the angle opposite edge $L$. Also, one can "go the other way", and calculate $\frac{\partial A}{\partial g_{12}}$ via

$$
\frac{\partial A}{\partial g_{12}}=\frac{\partial A}{\partial L} \frac{\partial L}{\partial L^{2}} \frac{\partial L^{2}}{\partial g_{12}}=\left(\frac{L}{2} \cot \theta_{o p}\right)\left(\frac{1}{2 L}\right)(-1)=-\frac{\cot \theta_{o p}}{4}
$$

which is consistent with Eq. (7.10), where we have used $L^{2}=g_{11}+g_{22}-g_{12}-g_{21}$ as well as $l_{1}^{2}=g_{11}$ and $l_{2}^{2}=g_{22}$. So, for each edge corresponding to a $G_{\gamma^{\prime}}^{\gamma}$, it is easiest to vary each of the attached hinges with respect to the metric none of whose diagonal elements corresponds to that edge.

Next we show that $G_{\gamma^{\prime}}^{\gamma}$ is proportional to $A \delta$ for $\gamma=\gamma^{\prime}$, and zero otherwise, independently of which edges were chosen to form the diagonal elements of the metric. So, the contribution from each hinge to the Einstein tensor for an edge is independent of the form of the metric if one is considering the one index up and one index down form of the Einstein tensor. So, Regge Calculus, to be "metric independent", would choose this form of the Einstein tensor to work with. In particular, this metric independence does not hold when considering $G_{\mu \nu}$ or $G^{\mu \nu}$. With a metric independent form one can have some sense of justification when one adds the various hinge contributions to edge L's $G_{L}^{L} ; G^{L L}$ and $G_{L L}$, not having metric independence, are undefined, or, at least, non-unique. Interestingly, the variation of $L$ contributes only to $G^{l_{1} l_{1}}$ and $G^{l_{2} l_{2}}$, but not $G^{L L} ; G^{L L}$ receives contributions from varying $l_{1}$ and $l_{2}$. In terms of the number of $G^{\mu}{ }_{\nu}$ 's, we are consistent: there would be three $g_{\rho \sigma}$ 's per triangle, giving three $G^{\mu}{ }_{\nu}$ 's per triangle, which we take to be $G^{l_{1}}{ }_{l_{1}}, G^{l_{2}}{ }_{l_{2}}$ and $G^{L}{ }_{L}$. 
A crucial question at this point is whether the form of the Einstein tensor, $L \cot \theta_{o p} / 2$, is metric independent. This form is obtained by varying with respect to edge length $L$, which is similar to varying with respect to edge length $L^{2}$ or $l_{1} \cdot l_{2}$. Hence, this form most closely corresponds to either $G^{L L}$ or $G^{12}$, and therefore is not metric independent. This can also be seen by noting our previous result of Eq. (7.12).

Next we calculate $G^{l_{1}}{ }_{l_{1}}$ and obtain

$$
G^{l_{1}}{ }_{l_{1}}=g_{11} G^{11}+g_{12} G^{12}=\left[l_{1}^{2}\left(\frac{g_{22}}{8 A}\right)+\left(l_{1} l_{2} \cos \theta_{o p}\right)\left(\frac{-l_{1} l_{2} \cos \theta_{o p}}{8 A}\right)\right] 2 \delta=A \delta
$$

which shows that our procedure is consistent.

\section{General Form of the Bianchi Identity}

In the previous sections on three-dimensional (Section 4) and four-dimensional (Section 5) rotations an exact and explicit form of the lattice Bianchi identity was given in terms of product of rotations along homotopically trivial paths. Later on in the paper an expression for the Riemann tensor and its dual were proposed, which among other properties, correctly reproduce the Regge field equations. In this section we will show that if one proceeds from the continuum Bianchi identities and uses the above given expression for the lattice Riemann tensor then the following is true. Firstly, the resulting lattice Bianchi identities coincide with their weak field counterparts discussed in the sections on 3-d and 4-d rotations. Secondly, when the infinitesimal form of the rotation matrix is promoted to the correct finite rotation expression (of Sections 4 and 5), the same exact identities derived previously are obtained. These result presented here are therefore intended to bring out one more time the close relationship between the Riemann tensor (in terms of which the continuum identities are naturally formulated) and the finite rotation matrices (in terms of which the exact lattice identities are formulated). A lattice expression for the Riemann tensor provides a natural bridge between these two different realms.

We will start here by considering the lattice Einstein tensor. Since the Einstein tensor's components live on edges, derivatives of the Einstein tensor must occur at vertices. Since the Einstein tensor is discontinuous at vertices, the easiest procedure is to integrate the Bianchi identities over a d-volume around a vertex and divide by that d-volume, at least in the case of small deficit angles. ${ }^{14}$ Then, the result is generalized to arbitrary deficit angles. So, we begin by deriving the

\footnotetext{
${ }^{14}$ The assumption of a flat coordinate system, so that edges have definite directions and so that we will be able to use Gauss' theorem, introduces errors of $\mathcal{O}\left(\delta^{2}\right)$.
} 
un-contracted, the partially contracted, and the completely contracted Bianchi identities in terms of the dual Riemann tensor, which will be the dual of the local Riemann curvature tensor as opposed to the global form, for reasons discussed earlier. In the continuum the un-contracted Bianchi identities read

$$
R_{\alpha \beta ; \gamma}^{\alpha^{\prime} \beta^{\prime}}+R_{\beta \gamma ; \alpha}^{\alpha^{\prime} \beta^{\prime}}+R_{\gamma \alpha ; \beta}^{\alpha^{\prime} \beta^{\prime}}=0
$$

The partially contracted and completely contracted Bianchi identities are obtained via contraction of $\alpha$ with $\alpha^{\prime}$ or/and $\beta$ with $\beta^{\prime}$. Using the d-dimensional dual Riemann tensor, one can write the un-contracted Bianchi identities into a divergence form via

$$
\begin{gathered}
\frac{1}{(d-2) !}\left[\left(\epsilon_{\alpha \beta \mu_{1} \ldots \mu_{d-2}} \tilde{R}^{\alpha^{\prime} \beta^{\prime} \mu_{1} \ldots \mu_{d-2}}\right)_{; \gamma}+\left(\epsilon_{\beta \gamma \nu_{1} \ldots \nu_{d-2}} \tilde{R}^{\alpha^{\prime} \beta^{\prime} \nu_{1} \ldots \nu_{d-2}}\right)_{; \alpha}+\left(\epsilon_{\gamma \alpha \sigma_{1} \ldots \sigma_{d-2}} \tilde{R}^{\alpha^{\prime} \beta^{\prime} \sigma_{1} \ldots \sigma_{d-2}}\right)_{; \beta}\right]=0 \\
\frac{1}{(d-3) !}\left[\left(\epsilon_{\alpha \beta \gamma \delta_{1} \ldots \delta_{d-3}} \tilde{R}^{\alpha^{\prime} \beta^{\prime} \gamma \delta_{1} \ldots \delta_{d-3}}\right)_{; \gamma}+\left(\epsilon_{\beta \gamma \alpha \delta_{1} \ldots \delta_{d-3}} \tilde{R}^{\alpha^{\prime} \beta^{\prime} \alpha \delta_{1} \ldots \delta_{d-3}}\right)_{; \alpha}+\left(\epsilon_{\gamma \alpha \beta \delta_{1} \ldots \delta_{d-3}} \tilde{R}^{\alpha^{\prime} \beta^{\prime} \beta \delta_{1} \ldots \delta_{d-3}}\right)_{; \beta}\right]=0 \\
\frac{1}{(d-3) !}\left(\epsilon_{\alpha \beta \gamma \delta_{1} \ldots \delta_{d-3}} \tilde{R}^{\alpha^{\prime} \beta^{\prime} \lambda \delta_{1} \ldots \delta_{d-3}}\right)_{; \lambda}=0
\end{gathered}
$$

Let us first consider the case of small deficit angles. The next step is (1) to integrate over and divide by ${ }^{d} V(v)$, the d-volume surrounding vertex $v,(2)$ to use Gauss' theorem, and (3) break up the (d-1)-dimensional surface integral into (d-1)-dimensional surfaces composed of (a) the 2-d Voronoi rotation areas and (b) the (d-3)-dimensional portions of the hinges' surface volumes (the ${ }^{d-3} V_{h}$ 's). One finds

$$
\frac{1}{(d-3) !{ }^{d} V(v)} \sum_{h \supset v} \int_{d-1} \epsilon_{\alpha \beta} V_{h} \delta_{1} \ldots \delta_{d-3} \tilde{R}^{\alpha^{\prime} \beta^{\prime} \lambda \delta_{1} \ldots \delta_{d-3}} \hat{n}_{\lambda} \sqrt{|g|} d^{d-1} x=0
$$

Rewriting $\tilde{R}$ using Eq. (6.7) and a generalized form of Eq. (6.10), letting the index $\lambda \rightarrow \lambda_{0}$, and defining the volume tensor

$$
V_{h}^{\rho_{0} \rho_{1} \ldots \rho_{d-3}} \equiv \frac{1}{(d-2) !} \sum_{\left(i_{0}, i_{1}, \ldots, i_{d-3}\right)=\mathcal{P}(0,1, \ldots, d-3)}(-1)^{\mathcal{P}} l_{h, i_{0}}^{\rho_{0}} l_{h, i_{1}}^{\rho_{1}} \ldots l_{h, i_{d-3}}^{\rho_{d-3}}
$$

where $(-1)^{\mathcal{P}}$ is the sign of the permutation, we find the integrand to be

$$
\epsilon_{\alpha \beta \gamma \delta_{1} \ldots \delta_{d-3}}\left[\frac{\delta_{h} U^{\alpha^{\prime} \beta^{\prime} d-2} V_{h}^{\lambda_{0} \delta_{1} \ldots \delta_{d-3}}}{A_{\Gamma}^{d-2} V_{h}}\right]\left[\frac{\epsilon_{\mu \nu \lambda_{0} \lambda_{1} \ldots \lambda_{d-3}} A_{\Gamma}^{\mu \nu}\left(l_{h, 1}-l_{h, 0}\right)^{\lambda_{1}} \ldots\left(l_{h, d-3}-l_{h, 0}\right)^{\lambda_{d-3}}}{A_{\Gamma}^{d-3} V_{h}}\right]
$$

Note that for each hinge the above expression is independent of the choice of assignment of the labels $l_{h, 0}, l_{h, 1}, \ldots, l_{h, d-3}$ to particular edges of the hinge. Furthermore when Eq. (8.6) is substituted into the above equation, each term in Eq. (8.6)'s sum can be replaced with $+l_{0}^{\lambda_{0}} l_{1}^{\delta_{1}} \ldots l_{d-3}^{\delta_{d-3}}$, so that 
the contraction of edges with the 2-nd Levi-Civita tensor always gives $\pm A_{\Gamma}{ }^{d-2} V_{h}$ where (4) we define $A_{\Gamma}^{\alpha \beta}$ such that the minus sign is always chosen (so as to be consistent with counter-clockwise rotations in 3-d). After canceling the integration with $A_{\Gamma}{ }^{d-3} V_{h}$, Eq. (8.5) can then be rewritten as

$$
-\frac{\epsilon_{\alpha \beta \gamma \delta_{1} \ldots \delta_{d-3}}}{(d-3) !{ }^{d} V(v)} \sum_{h \supset v} \delta_{h} U^{\alpha^{\prime} \beta^{\prime}} \sum_{h_{b} \subset h} l_{h, 1}^{\delta_{1}} \ldots l_{h, d-3}^{\delta_{d-3}}=0
$$

where $h_{b}$ refers to a "hinge base", and is a (d-3)-simplex, and hence necessarily a "base" to the hinges containing it. One can then invert the sums to find

$$
-\frac{\epsilon_{\alpha \beta \gamma \delta_{1} \ldots \delta_{d-3}}}{(d-3) !{ }^{d} V(v)} \sum_{h_{b} \supset v}\left(\sum_{h \supset h_{b}} \delta_{h} U_{h, h_{b}}^{\alpha^{\prime} \beta^{\prime}}\right) l_{h_{b}, 1}^{\delta_{1}} \ldots l_{h_{b}, d-3}^{\delta_{d-3}}=0
$$

where a unique labeling assignment has been chosen for each hinge base, Here $U_{h, h_{b}}^{\alpha^{\prime} \beta^{\prime}}= \pm U^{\alpha^{\prime} \beta^{\prime}}$, the plus or minus sign chosen when $\left\{l_{h b, 1}, \ldots, l_{h_{b}, d-3}\right\}$ is, respectively, an even or odd permutation of $\left\{l_{h, 1}, \ldots, l_{h, d-3}\right\}$. I.e., the sign of $U_{h, h_{b}}^{\alpha^{\prime} \beta^{\prime}}$ is chosen such that $\epsilon_{\alpha \beta \gamma \delta_{1} \ldots \delta_{d-3}} U_{h, h_{b}}^{\alpha \beta} l_{0}^{\gamma} l_{h_{b}, 1}^{\delta_{1}} \ldots l_{h_{b}, d-3}^{\delta_{d-3}}<0$ for one, and hence for all via projection to $3-\mathrm{d}, h \supset h_{b}$. ${ }^{15}$

Using as the equation for $V_{h_{b}}^{\delta_{1} \ldots \delta_{d-3}}$ a (d-3)-dimensional version of Eq. (8.6), the above equation can then be rewritten as

$$
-\frac{\epsilon_{\alpha \beta \gamma \delta_{1} \ldots \delta_{d-3}}}{{ }^{d} V(v)} \sum_{h_{b} \supset v}\left(\sum_{h \supset h_{b}} \delta_{h} U_{h, h_{b}}^{\alpha^{\prime} \beta^{\prime}}\right) V_{h_{b}}^{\delta_{1} \ldots \delta_{d-3}}=0
$$

which for the special cases of three and four dimensions reduces to the approximate small deficit angle expressions discussed previously in the sections on 3d (Section 4) and 4d (Section 5) rotations. Using $\sum \delta_{h} U^{\alpha^{\prime} \beta^{\prime}} \approx \sum(\operatorname{Rot}-1)^{\alpha^{\prime} \beta^{\prime}} \approx\{\Pi[1+(\operatorname{Rot}-1)]-1\}^{\alpha^{\prime} \beta^{\prime}}=[\Pi(R o t)-1]^{\alpha^{\prime} \beta^{\prime}}$, where “ $\approx$ " symbolizes equalities to first order in deficit angles, the above equation generalizes to, for arbitrary deficit angles, ${ }^{16}$

$$
-\frac{\epsilon_{\alpha \beta \gamma \delta_{1} \ldots \delta_{d-3}}}{{ }^{d} V(v)} \sum_{h_{b} \supset v}\left[\prod_{h \supset h_{b}}\left(\operatorname{Rot}_{h, h_{b}}\right)-1\right]^{\alpha^{\prime} \beta^{\prime}} V_{h_{b}}^{\delta_{1} \ldots \delta_{d-3}}=0
$$

where the product of rotations is over a null path, and where $\beta^{\prime}$ and $\alpha^{\prime}$ refer to, respectively, the first index of the first rotation matrix and the last index of the last rotation matrix. In the special case of three and four dimensions the above result is equivalent to the exact expressions discussed previously in the sections on $3 \mathrm{~d}$ (Section 4$)$ and $4 \mathrm{~d}$ (Section 5) rotations. ${ }^{17}$

\footnotetext{
${ }^{15}$ Here, we replace $A_{\Gamma}^{\alpha^{\prime} \beta^{\prime}}$ with $A_{\Gamma, h_{b}}^{\alpha^{\prime} \beta^{\prime}} \propto+U_{h, h_{b}}^{\alpha^{\prime}} \beta^{\prime}$.

${ }^{16}$ Here, $\prod(R o t)$ is a product of mixed tenors with the first index up and the second down; however the first index of the last rotation matrix (on the left), $\alpha^{\prime}$, and the 2 nd index of the first rotation matrix, $\beta^{\prime}$, can be either up or down.

${ }^{17}$ In the Lorentzian case, the above equations have a plus sign; each term of the above equation gets an additional minus sign either because of the reason noted in the footnote to Eq. (6.7) or because the presence of the time component in a hinge would otherwise cause a reversal of the direction (and sign) of $A_{\Gamma, h_{b}}^{\alpha^{\prime} \beta^{\prime}}$ due to the change in sign of $\epsilon_{\alpha \beta \gamma \delta_{1} \ldots \delta_{d-3}} U_{h, h_{b}}^{\alpha \beta} V_{h}^{\gamma \delta_{1} \ldots \delta_{d-3}}$.
} 
Let us add a few comments regarding the result just obtained. The coordinate system in which this equation is written is one of the possible global coordinate systems described in the section on Geometric Setups (Section 3). By taking the Levi-Civita tensor out of the $\sum_{h_{b} \supset v}$, we are taking the Levi-Civita tensor to be defined at vertices such as $v .{ }^{18}$ Now, one can ask how we can use a d-dimensional Levi-Civita tensor when in our derivation of $\mathcal{G}_{l}{ }^{l}$ we only used $(d-2) \times(d-2)$ metrics; the answer is that there we were considering Voronoi volumes which had two dimensions not set by edge lengths, whereas here all dimensions of every volume emanating from vertex $v$ involve edge lengths.

Also, the partially and fully contracted Bianchi identities, now for arbitrary deficit angles, are easily obtained via contractions. The above Bianchi identities can be converted to scalar form via dot products with $d$ d-vectors which form a d-volume in our going around coordinate system discussed in Section 3. Do note that, when one changes going around coordinate systems, dot products do change, so that even here, what is a Bianchi identity for one going around coordinate system may not be for another going around coordinate system. Another (going around) coordinate system difference would be that $\Gamma$ 's such as that in Eq. (3.4) would be unity for some coordinate systems, and not for others, so that a different order of rotations, utilizing the $\Gamma(\mathrm{s})$ which are unity, would be appropriate for these other coordinate systems. Still, once the dot products are taken, the Bianchi identities are in terms of edge lengths, which are independent of coordinate system; hence, once dot products are taken, any Bianchi identity is true independent of the going around coordinate system. In summary, each going around coordinate system has its own Bianchi identities, which themselves can be projected into coordinate system independent equalities. Of course, once one substitutes in for the deficit angles their explicit expression in terms of edge lengths, one attains zero identically. However, if one does not do this, these identities give relations between the deficit angles and squared edge lengths.

\section{Bianchi Identities in Four Dimensions}

The discussion of the previous section was for a general dimension $d$. In this section we will focus on the four-dimensional case, and proceed from the continuum Bianchi identities to derive the weak field expression for the lattice Bianchi identities. Once the resulting infinitesimal rotations are promoted to finite lattice rotations, the resulting identities coincide with the exact form given

\footnotetext{
${ }^{18}$ We needn't have done this; however, taking it out of $\sum_{h \supset h_{b}}$ or $\prod_{h \supset h_{b}}$ avoids unnecessary complications.
} 
previously (Section 5). At the end of this section we will then collect the explicit formulae for the exact un-contracted, partially contracted, and fully contracted lattice Bianchi identity, valid for arbitrary manifolds.

In the four-dimensional case the un-contracted identity in the continuum reads

$$
\frac{1}{2}\left[\left(\epsilon_{\alpha \beta \mu \nu} \tilde{R}^{\alpha^{\prime} \beta^{\prime} \mu \nu}\right)_{; \gamma}+\left(\epsilon_{\beta \gamma \mu \nu} \tilde{R}^{\alpha^{\prime} \beta^{\prime} \mu \nu}\right)_{; \alpha}+\left(\epsilon_{\gamma \alpha \mu \nu} \tilde{R}^{\alpha^{\prime} \beta^{\prime} \mu \nu}\right)_{; \beta}\right]=0
$$

Now, defining the index $\delta$ such that $[\alpha \beta \gamma \delta] \neq 0$ (for $d>4$, we choose one set of indices, an ordered set, $\vec{\delta} \equiv \delta_{1} \delta_{2} \ldots \delta_{d-3}$, which satisfies $\left.[\alpha \beta \gamma \vec{\delta}] \neq 0\right)$, we find

$$
\left(\epsilon_{\alpha \beta \gamma \delta} \tilde{R}^{\alpha^{\prime} \beta^{\prime} \gamma \delta}\right)_{; \gamma}+\left(\epsilon_{\beta \gamma \alpha \delta} \tilde{R}^{\alpha^{\prime} \beta^{\prime} \alpha \delta}\right)_{; \alpha}+\left(\epsilon_{\gamma \alpha \beta \delta} \tilde{R}^{\alpha^{\prime} \beta^{\prime} \beta \delta}\right)_{; \beta}=0
$$

where, as we have stated, $\alpha, \beta, \gamma$ and $\delta$ are not summed anywhere in the equation. We can now rewrite the above equation as

$$
\left(\epsilon_{\alpha \beta \gamma \delta} \tilde{R}^{\alpha^{\prime} \beta^{\prime} \lambda \delta}\right)_{; \lambda}=0
$$

where $\lambda$ is summed over, and $\delta$ is not. Actually, in $4-\mathrm{d}$, the above equation does not change even if $\delta$ is summed over all directions; for $d>4$, one simply multiplies by a factor of $1 /(d-3)$ ! to get the exact correspondence to the un-contracted Bianchi identities if one decides to sum on $\delta$ (which, for $d>4$, becomes $\vec{\delta}$, and the "sum on $\delta$ " is the sum over all the $\delta$ 's of $\vec{\delta}$, which has non-zero terms only when none of the $\delta$ 's are equal to $\alpha, \beta$ or $\gamma$ ).

To get the partially contracted Bianchi identites, one simply either sets $\alpha=\alpha^{\prime}$ or $\beta=\beta^{\prime}$, and then sums over either $\alpha$ or $\beta$. Setting $\beta=\beta^{\prime}$ gives

$$
\left(\epsilon_{\alpha \beta \gamma \delta} \tilde{R}^{\alpha^{\prime} \beta \lambda \delta}\right)_{; \lambda}=0
$$

Here, we use the Einstein summation convention for all indices, so that for $d>4$, we would need a factor of $1 /(d-3)$ ! from Eq. (9.3). In particular, we note that $\delta$ must be summed over, because we are summing over two values of $\beta$, and therefore $\delta$ must run over different indices (or, for $d>4$, different $\beta$ 's implies that not all $\vec{\delta}$ 's which give non-zero terms will be comprised of the same set of indices). To get the fully contracted Bianchi identities, we set $\alpha=\alpha^{\prime}$ and sum over $\alpha$ in the partially contracted Bianchi identities to find

$$
\left(\epsilon_{\alpha \beta \gamma \delta} \tilde{R}^{\alpha \beta \lambda \delta}\right)_{; \lambda}=0
$$

where, once again, we would have the factor of $1 /(d-3)$ ! for $d>4$.

The next step is to interpret the un-contracted Bianchi identities, Eq. (9.3) in Regge Calculus. Using the lattice expression for the dual of the Riemann curvature tensor, Eq. (6.22), we have 


$$
\frac{1}{6}\left(\frac{\epsilon_{\alpha \beta \gamma \delta}}{{ }^{4} V_{h}} \delta_{h} U^{\alpha^{\prime} \beta^{\prime}} A_{h}^{\lambda \delta}\right)_{; \lambda}=0
$$

We now integrate this over a vertex $v$, and divide by the sum of all Voronoi four-volumes surrounding that vertex, ${ }^{4} V_{v}$.

$$
\frac{1}{6} \frac{1}{{ }^{4} V_{v}} \int_{v}\left(\frac{\epsilon_{\alpha \beta \gamma \delta}}{{ }^{4} V_{h}} \delta_{h} U^{\alpha^{\prime} \beta^{\prime}} A_{h}^{\lambda \delta}\right)_{; \lambda} \sqrt{|g|} d^{4} x=0
$$

One can rewrite this in terms of edges, as the components of the Einstein tensor live on edges, and because the completely contracted Bianchi identities are written in terms of components of the Einstein tensor.

$$
\frac{1}{6} \frac{1}{{ }^{4} V_{v}} \sum_{h \supset v} \int_{h}\left(\frac{\epsilon_{\alpha \beta \gamma \delta}}{{ }^{4} V_{h}} \delta_{h} U^{\alpha^{\prime} \beta^{\prime}} A_{h}^{\lambda \delta}\right)_{; \lambda} \sqrt{|g|} d^{4} x=0
$$

Since each hinge appears on two edges, we find

$$
\frac{1}{2 \cdot 6} \frac{1}{{ }^{4} V_{v}} \sum_{l \supset v} \sum_{h \supset l} \int_{h}\left(\frac{\epsilon_{\alpha \beta \gamma \delta}}{{ }^{4} V_{h}} \delta_{h} U^{\alpha^{\prime} \beta^{\prime}} A_{h}^{\lambda \delta}\right)_{; \lambda} \sqrt{|g|} d^{4} x=0
$$

which implies that the dual Riemann tensor for an edge is one half of the sum of the dual Riemann tensor for all hinges containing that edge. Using Gauss' theorem, one then finds

$$
\frac{1}{6} \frac{1}{{ }^{4} V_{v}} \sum_{l \supset v} \sum_{h \supset l} \int_{h}\left(\frac{\epsilon_{\alpha \beta \gamma \delta}}{{ }^{4} V_{h}} \delta_{h} U^{\alpha^{\prime} \beta^{\prime}} A_{h}^{\lambda \delta}\right) \hat{n}_{\lambda} \sqrt{|g|} d^{3} x=0
$$

Noting that the radial direction is contained within the hinge, one can integrate over the loop area, leaving the only integration variable to be the direction $\pm(L-l)$, where $L$ is the other edge of the hinge which also contains (and is directed outward from) the vertex $v . L-l$ here is the unique direction, up to a sign, perpendicular to the surface normal without any component contained within the loop area. We choose $+(L-l)$ to ensure consistency throughout the sums. One has therefore

$$
\frac{1}{6} \frac{1}{{ }^{4} V_{v}} \sum_{l \supset v} \sum_{h \supset l} \int_{h}\left(A_{\Gamma} \frac{\epsilon_{\alpha \beta \gamma \delta}}{{ }^{4} V_{h}} \delta_{h} U^{\alpha^{\prime} \beta^{\prime}} A_{h}^{\lambda \delta}\right) \hat{n}_{\lambda} \sqrt{|g|} d s=0
$$

Noting that $A_{h}^{\lambda \delta}=L^{\lambda} l^{\delta}-l^{\lambda} L^{\delta}$ (where we have chosen a sign convention for $A_{h}$ ), one can write the dot product $L \cdot \hat{n}$ as

$$
\hat{n} \cdot L=\frac{\left[\epsilon_{\mu \nu \lambda \sigma} A_{\Gamma}^{\mu \nu}(l-L)^{\sigma}\right] L^{\lambda}}{A_{\Gamma}|L-l|}=\frac{\epsilon_{\mu \nu \lambda \sigma} A_{\Gamma}^{\mu \nu} l^{\sigma} L^{\lambda}}{A_{\Gamma}|L-l|}=\frac{ \pm 2 A_{h}}{|L-l|}
$$

Then using this result and a similar one for $l \cdot \hat{n}$ one obtains 


$$
\frac{1}{6} \frac{1}{{ }^{4} V_{v}} \sum_{l \supset v} \sum_{h \supset l} \frac{\epsilon_{\alpha \beta \gamma \delta}}{{ }^{4} V_{h}} \delta_{h} U^{\alpha^{\prime} \beta^{\prime}} A_{\Gamma} A_{h}(l-L)^{\delta}=0
$$

which is equivalent to

$$
\frac{1}{{ }^{4} V_{v}} \sum_{l \supset v} \epsilon_{\alpha \beta \gamma \delta}( \pm) \sum_{h \supset l} \delta_{h} U^{\alpha^{\prime} \beta^{\prime}}(l-L)^{\delta}=0
$$

where the \pm sign will indicate whether the hinges, for each particular hinge base, are gone around in a counter-clockwise or clockwise fashion. This is then further simplified when one considers that the contribution for each edge is the same whether it be in its $L$ (non-hinge base) contribution, or its $l$ (hinge base) contribution, noting that $l \leftrightarrow L$ leads to $\pm \rightarrow \mp$. Indeed, given that we started with a sum over hinges, Eq. (9.8), and broke that down into a sum over edges, Eq. (9.9), each edge's contribution to the hinge must be identical. So, we can take each edge's $l$ contribution, double it, and omit its $L$ contribution to find for small deficit angle

$$
\frac{1}{{ }^{4} V_{v}} \sum_{l \supset v} \epsilon_{\alpha \beta \gamma \delta}( \pm) \sum_{h \supset l} \delta_{h} U^{\alpha^{\prime} \beta^{\prime}} l^{\delta}=0
$$

The partially contracted and completely contracted forms are then easy to obtain. The partially contracted form can be written for small deficit angle as either

$$
\frac{1}{{ }^{4} V_{v}} \sum_{l \supset v} \sum_{h \supset l} \epsilon_{\alpha \beta \gamma \delta} \delta_{h} U^{\alpha^{\prime} \beta}(L-l)^{\delta}=0
$$

or

$$
\frac{1}{{ }^{4} V_{v}} \sum_{l \supset v} \sum_{h \supset l} \epsilon_{\alpha \beta \gamma \delta} \delta_{h} U^{\alpha \beta^{\prime}}(L-l)^{\delta}=0
$$

and the fully contracted form for small deficit angle is

$$
\frac{1}{{ }^{4} V_{v}} \sum_{l \supset v} \sum_{h \supset l} \epsilon_{\alpha \beta \gamma \delta} \delta_{h} U^{\alpha \beta}(L-l)^{\delta}=0
$$

We note that each fixed $l$ term in $\sum_{l}$ can be taken to correspond to one term in (what was) the divergence, because each edge length corresponds to one component of the Einstein tensor $G^{\lambda}$.

Next we note that these small deficit angle forms can all be rewritten in terms of rotation matrices. For example, the un-contracted form can be written as

$$
\frac{1}{{ }^{4} V_{v}} \sum_{l \supset v} \sum_{h \supset l} \epsilon_{\alpha \beta \gamma \delta}\left(\operatorname{Rot}_{h}-1\right)^{\alpha^{\prime} \beta^{\prime}}(L-l)^{\delta}=0
$$

and for arbitrary deficit angles, this generalizes to

$$
\frac{1}{{ }^{4} V_{v}} \sum_{l \supset v} \epsilon_{\alpha \beta \gamma \delta}\left[\prod_{h \supset l}\left(\operatorname{Rot}_{h}\right)-1\right]^{\alpha^{\prime} \beta^{\prime}}(L-l)^{\delta}=0
$$


where $\beta^{\prime}$ refers to the second index of the first rotation matrix, and $\alpha^{\prime}$ refers to the first index of the last rotation matrix. The above expression coincides with the exact result obtained previously in the section on finite rotations in four dimensions (Section 5). The partially contracted Bianchi identities can then be written for arbitrary deficit angles as either

$$
\frac{1}{{ }^{4} V_{v}} \sum_{l \supset v} \epsilon_{\alpha \beta \gamma \delta}\left[\prod_{h \supset l}\left(\operatorname{Rot}_{h}\right)-1\right]^{\alpha^{\prime} \beta}(L-l)^{\delta}=0
$$

or

$$
\frac{1}{{ }^{4} V_{v}} \sum_{l \supset v} \epsilon_{\alpha \beta \gamma \delta}\left[\prod_{h \supset l}\left(\operatorname{Rot}_{h}\right)-1\right]^{\alpha \beta^{\prime}}(L-l)^{\delta}=0
$$

depending on the choice of indices. Finally the completely contracted Bianchi identities can be written for arbitrary deficit angle as

$$
\frac{1}{{ }^{4} V_{v}} \sum_{l \supset v} \epsilon_{\alpha \beta \gamma \delta}\left[\prod_{h \supset l}\left(\operatorname{Rot}_{h}\right)-1\right]^{\alpha \beta}(L-l)^{\delta}=0
$$

We can see that this has the correct units, $1 /\left({ }^{3} V\right)$, by taking the metric, and hence the Levi=Civita tensor, to be dimensionless.

\section{Bianchi Identities in Three Dimensions}

Let us repeat the above construction in three dimensions, without going through the details which very much parallel what was done in the four dimensional case just discussed. For $d=3$, since the hinges are the edges, we get for the completely un-contracted Bianchi identities, valid for arbitrary deficit angles,

$$
\frac{1}{{ }^{3} V_{v}} \epsilon_{\alpha \beta \gamma}\left[\prod_{l}\left(\operatorname{Rot}_{l}\right)-1\right]^{\alpha^{\prime} \beta^{\prime}}=0
$$

Then the 3-d partially contracted Bianchi identites for arbitrary deficit angles can be written as either

$$
\frac{1}{{ }^{3} V_{v}} \epsilon_{\alpha \beta \gamma}\left[\prod_{l}\left(\operatorname{Rot}_{l}\right)-1\right]^{\alpha^{\prime} \beta}=0
$$

or

$$
\frac{1}{{ }^{3} V_{v}} \epsilon_{\alpha \beta \gamma}\left[\prod_{l}\left(\operatorname{Rot}_{l}\right)-1\right]^{\alpha \beta^{\prime}}=0
$$

and finally the 3-d completely contracted Bianchi identities for arbitrary deficit angles read

$$
\frac{1}{{ }^{3} V_{v}} \epsilon_{\alpha \beta \gamma}\left[\prod_{l}\left(\operatorname{Rot}_{l}\right)-1\right]^{\alpha \beta}=0
$$


These expressions coincide with the exact result obtained previously in the section on finite rotations in three dimensions (Section 4). In three dimensions and for small deficit angles these results reduce to

$$
\frac{1}{{ }^{3} V_{v}} \sum_{l \supset v} \epsilon_{\alpha \beta \gamma} \delta_{l} U^{\alpha^{\prime} \beta^{\prime}}=0
$$

The 3-d partially contracted Bianchi identites can be then be written, again for small deficit angles, as either

$$
\frac{1}{{ }^{3} V_{v}} \sum_{l \supset v} \epsilon_{\alpha \beta \gamma} \delta_{l} U^{\alpha^{\prime} \beta}=0
$$

or

$$
\frac{1}{{ }^{3} V_{v}} \sum_{l \supset v} \epsilon_{\alpha \beta \gamma} \delta_{l} U^{\alpha \beta^{\prime}}=0
$$

depending on the choice of indices. Finally for small deficit angles the 3-d completely contracted Bianchi identities are

$$
\frac{1}{{ }^{3} V_{v}} \epsilon_{\alpha \beta \gamma} \sum_{l \supset v} \delta_{l} U^{\alpha \beta}=0
$$

\section{Bianchi Identities in Dimensions Greater than Four}

For dimensions $d>4$, our $4-d$ results are generalized after recalling that, in the original integration, each hinge appears once, so that each hinge should appear once in our results as well. For each hinge, all of its $(d-2)$ edges bordering vertex $v$ appear in $\sum_{l}$ once, so we need to divide

by $(d-2)$. Also, for a fixed edge contained within a hinge, there are $\left(\begin{array}{c}d-3 \\ d-4\end{array}\right)=d-3$ hinge bases ((d-3)-dimensional simplexes which are labeled by $\left.h_{b}\right)$, which are summed over which that hinge contains. So, we must also divide by $(d-3)$. For convenience, the order of $L_{1}, L_{2}, \ldots, L_{d-3}$ can be chosen such that $\epsilon_{\alpha \beta \gamma \vec{\delta}} \operatorname{Rot}_{h}^{\alpha \beta} l^{\gamma}(L-l)^{\vec{\delta}}>0$. Therefore for $d>4$ the un-contracted Bianchi identities for small deficit angles read

$$
\frac{1}{(d-2)(d-3)} \frac{1}{d} V_{v} \sum_{l \supset v} \sum_{h_{b} \supset l} \epsilon_{\alpha \beta \gamma \vec{\delta}}\left[\sum_{h=\left(h_{b}, L\right)} \delta_{h} U^{\alpha \beta}\left(l_{1}-l\right)^{\delta_{1}}\left(l_{2}-l\right)^{\delta_{2}} \ldots\left(l_{d-4}-l\right)^{\delta_{d-4}}(l-L)^{\delta_{d-3}}=0\right.
$$

where the last sum can be viewed either as over $h \supset h_{b}$ or over edges $L$ which, along with $h_{b}$, form a hinge. Taking the latter view, we can note that, for a fixed term in this triple sum, we should get the same term when $l \leftrightarrow L$. This, indeed, is the reason we were able to divide by $(d-2)$ in Eq. (11.1). So one can simply double the above result and let $(l-L)^{\delta_{d-3}} \rightarrow l_{d-3}^{\delta}$, which dramatically 
simplifies the answer to

$$
\frac{2}{(d-2)(d-3)} \frac{1}{d V_{v}} \sum_{l \supset v} \sum_{h_{b} \supset l} \epsilon_{\alpha \beta \gamma \vec{\delta}}\left[\sum_{h=\left(h_{b}, L\right)} \delta_{h} U^{\alpha \beta} l_{1}^{\delta_{1}} l_{2}^{\delta_{2}} \ldots l_{d-4}^{\delta_{d-4}} l^{\delta_{d-3}}=0\right.
$$

Now, as opposed to $\frac{1}{(d-2)(d-3)} \sum_{l \supset v} \sum_{h_{b} \supset l}$ we could have written just $\frac{1}{d-2} \sum_{h_{b} \supset v}$, but this would not explicitly involve the edge lengths. And, a sum over edge lengths is most directly related to a divergence because, as mentioned earlier, each fixed $l$ term in $\sum_{l}$ can be taken to correspond to one term in (what was) the divergence, because each edge length corresponds to one component of the Einstein tensor. Additionally, the identities would not look as neat, as there would not be a natural edge to subtract from the other hinge edges to get a hinge's $(d-3)$-surface volume.

The above formulas were for small deficit angles. For arbitrary deficit angles, we find the un-contracted Bianchi identities to be

$$
\frac{1}{(d-2)(d-3)} \frac{1}{d V_{v}} \sum_{l \supset v} \sum_{h_{b} \supset l} \epsilon_{\alpha \beta \gamma \vec{\delta}}\left[\left(\prod_{h \supset h_{b}} \operatorname{Rot}_{h}\right)-1\right]^{\alpha^{\prime} \beta^{\prime}}(L-l)_{h_{b}, v}^{\vec{\delta}}=0
$$

The partially contracted Bianchi identities can be written as either

$$
\frac{1}{(d-2)(d-3)} \frac{1}{d V_{v}} \sum_{l \supset v} \sum_{h_{b} \supset l} \epsilon_{\alpha \beta \gamma \vec{\delta}}\left[\left(\prod_{h \supset h_{b}} R_{0} t_{h}\right)-1\right]^{\alpha^{\prime} \beta}(L-l)_{h_{b}, v}^{\vec{\delta}}=0
$$

or

$$
\frac{1}{(d-2)(d-3)} \frac{1}{d} V_{v} \sum_{l \supset v} \sum_{h_{b} \supset l} \epsilon_{\alpha \beta \gamma \vec{\delta}}\left[\left(\prod_{h \supset h_{b}} \operatorname{Rot}_{h}\right)-1\right]^{\alpha \beta^{\prime}}(L-l)_{h_{b}, v}^{\vec{\delta}}=0
$$

depending on the choice of indices, and finally the completely contracted Bianchi identities can be written as

$$
\frac{1}{(d-2)(d-3)} \frac{1}{d} V_{v} \sum_{l \supset v} \sum_{h_{b} \supset l} \epsilon_{\alpha \beta \gamma \vec{\delta}}\left[\left(\prod_{h \supset h_{b}} \operatorname{Rot}_{h}\right)-1\right]^{\alpha \beta}(L-l)_{h_{b}, v}^{\vec{\delta}}=0
$$

\section{Conclusions}

In this paper we have derived an exact form for the Bianchi identities in simplicial gravity. In four dimensions these are given by Eqs. (9.20), (9.21) and (9.23) for the un-contracted, partially contracted and fully contracted form respectively. In three dimensions the corresponding expressions are given by Eqs. (10.1), (10.2) and (10.4), while above four dimension the corresponding general results are in Eqs. (11.3), (11.4) and (11.6), with an alternative but equivalent form of the un-contracted Bianchi identity given in Eq. (8.11). The explicit form of the rotation matrices 
appearing in the above-quoted exact Bianchi identities was constructed explicitly and presented in Sections 4 (three dimensional case) and 5 (four dimensional case).

While fairly unwieldy in their explicit form, these identities can be shown to reduce to their known weak field expression in the limit of small curvatures. They provide an explicit, local relationship between deficit angles belonging to neighboring simplices. Their existence can be viewed as a consequence of the local invariance of the Regge action under small gauge deformations of edge lengths emanating from a vertex, just as the continuum Bianchi identity can be derived from the local gauge invariance of the gravitational action.

The relationship between the lattice Bianchi identities and the Regge lattice equations of motion has been investigated as well. In the continuum the contracted Bianchi identities ensure the consistency of the gravitational field equations. One would expect that the same should be true on the lattice, in the sense that a lattice "covariant divergence" of the lattice field equations would identically yield zero, as a consequence of the lattice Bianchi identity. We have shown that that is indeed the case in the lattice theory.

When in the Regge lattice case we go from the discrete Einstein-Hilbert action and its equations of motion to the fully contracted Bianchi identities, and then write down an explicit form for the partially contracted and un-contracted Bianchi identities as well, we find that the relation between the three types of Bianchi identities in the Regge theory is basically simply the contraction of indices, as in the continuum. Also, as the product of rotation matrices around a null path is critical in understanding the form of all three types of Bianchi identities, we have provided both a quick way to calculate this product and an understanding of, in particular, its antisymmetric components. Finally by appropriately projecting the lattice Bianchi identities, we have derived explicit expressions depending on edge lengths squared only.

All of this is exact, valid for arbitrary deficit angles, though we have provided first order approximations to our results for the product of rotation matrices. An exact form for the lattice Bianchi identity should be useful in a variety of context, including numerical schemes for classical and quantum gravity. In the classical case, the accuracy of four-dimensional time evolution codes could be checked by evaluating the Bianchi identity along a time evolved trajectory.

\section{Acknowledgements}

The authors wish to thank J. Hartle and R.M. Williams for very useful discussions on the subject of this paper. 


\section{References}

[1] T. Regge, Nuovo Cimento 19558 (1961).

[2] Wheeler, J. A., 'Geometrodynamics and the Issue of the Final State.'. In Relativity, Groups, and Topology (Ed. C. DeWitt and B. S. DeWitt). New York: Gordon and Breach, pp. 467-500, 1964 .

[3] M. Roček and R. M. Williams, Phys. Lett. 104B 31 (1981); Z. Phys. C21 371 (1984).

[4] M. Roček and R. M. Williams, 'Introduction to quantum Regge calculus', in Quantum Structure of Space and Time, edited by M. J. Duff and C. J. Isham (Cambridge University Press, 1982).

[5] J. Cheeger, W. Müller and R. Schrader, Comm. Math. Phys. 92405 (1984).

[6] J. Cheeger, W. Müller and R. Schrader, in 'Unified Theories Of Elementary Particles', Heisenberg Symposium, München 1981, Springer Lecture Notes in Physics, P. Breitlohner and H.P. Dürr eds., New York 1982.

[7] H. W. Hamber and R. M. Williams, Nucl. Phys. B248 392 (1984); B260 747 (1985); Phys. Lett. 157B 368 (1985); Nucl. Phys. B267 482 (1986); B269 712 (1986).

[8] J. B. Hartle, J. Math. Phys. 26 (1985) 804; 27 (1985) 287; 30 (1989) 452.

[9] J. B. Hartle, in the Proceedings of the Third Moscow Seminar on Quantum Gravity, M. Markov, V. Berezin and V. Frolov eds., World Scientific 1985.

[10] H. W. Hamber, 'Simplicial Quantum Gravity', in Critical Phenomena, Random Systems, Gauge Theories, 1984 Les Houches Summer School, Session XLIII, edited by K. Osterwalder and R. Stora (North Holland, Amsterdam, 1986); Nucl. Phys. Proc. Suppl. 25A (1992) 150175 .

[11] R. Friedberg and T. D. Lee, Nucl. Phys. B242 (1984) 145;

G. Feinberg, R. Friedberg, T. D. Lee and H. C. Ren, Nucl. Phys. B245 (1984) 343.

[12] T. D. Lee, in 'Discrete Mechanics', 1983 Erice International School of Subnuclear Physics, vol. 21 (Plenum Press, New York 1985).

[13] H. W. Hamber, in 'Probabilistic Methods in Quantum Field Theory and Quantum Gravity', (Cargèse 1989, published by Plenum Press, New York 1990) pp. 243-257. 
[14] R. M. Williams, 'Discrete Quantum Gravity: the Regge Calculus Approach', Int. J. Mod. Phys. B 6, 2097-2108, 1992.

[15] J. Fröhlich, 'Regge Calculus and Discretized Gravitational Functional Integrals', I.H.E.S. preprint 1981, published in Non-Perturbative Quantum Field Theory: Mathematical Aspects and Applications, Selected Papers. Singapore: World Scientific, pp. 523-545, 1992.

[16] T. Regge and R. M. Williams, J. Math. Phys. 41, 3964 (2000) [gr-qc/0012035].

[17] R. M. Williams, Nucl. Phys. Proc. Suppl. 57, 73 (1997) [gr-qc/9702006].

[18] R. P. Feynman, Lectures on Gravitation, edited by F. Morinigo and W. Wagner, (Caltech lecture notes, 1962).

[19] S. Weinberg, 'Gravitation and Cosmology', (Wiley, New York 1972).

[20] C. W. Misner, K. S. Thorne and J. A. Wheeler, 'Gravitation', Freeman, San Francisco 1973.

[21] R. M. Wald, 'General Relativity', University of Chicago Press, 1984.

[22] W. Miller, Found. of Phys., Vol 16, No. 2, (1986); Class. Quant. Grav. 14 (1997) L199-L204.

[23] P. Morse, Journal Class. Quant. Grav. 9 (1992) 2489.

[24] C. Y. Wong, J. Math. Phys. 12 (1971) 70;

P. A. Collins and R. M. Williams, Phys. Rev. D5 (1972) 1908; D7 (1973) 965; D10 (1974) 353 ;

R. M. Williams and G. F. R. Ellis, Gen. Rel. Grav. 13 (1981) 361.

[25] R. Sorkin, Phys. Rev. D 12, 385-396, 1975; J. Math. Phys. 16 (1975) 2432 and 19 (1978) 1800 .

[26] A. Gentle, D. Holz, W. A. Miller, and J. A. Wheeler, Class. Quant. Grav. 16 (1999) 1979;

S. Chakrabarti, A. Gentle, A. Kheyfets and W. A. Miller, Class. Quant. Grav. 16 (1999) 2381;

W. A. Miller, Class. Quant. Grav. 14 (1997) L199;

A. Gentle and W. A. Miller, Class. Quant. Grav. 15 (1998) 389;

J. W. Barrett, M. Galassi, W. A. Miller, R. D. Sorkin, P. A. Tuckey and R. M. Williams, Int. J. Theor. Phys. 36 (1997) 815.

[27] R.M. Williams and P.A. Tuckey, Class. Quant. Grav. 9 (1992) 1409;

R.M. Williams, Int. J. Mod. Phys. B6 (1992) 2097. 
[28] M. Rocek and R. M. Williams, Class. Quant. Grav. 2, 701 (1985).

[29] B. Berg, Phys. Rev. Lett. 55904 (1985); Phys. Lett. B176 39 (1986).

[30] R. M. Williams, Class. Quant. Grav. 3, 853 (1986).

[31] T. Piran and R. M. Williams, Phys. Rev. D 33, 1622 (1986).

[32] A. Jevicki and M. Ninomiya, Phys. Lett. B 150, 115 (1985); Phys. Rev. D 33, 1634 (1986).

[33] M. Bander, Phys. Rev. Lett. 57, 1825 (1986).

[34] M. Roček and R. M. Williams, Phys. Lett. B273, 95 (1991).

[35] H.W. Hamber and R.M. Williams, Phys. Rev. D47 (1993) 510.

[36] G. Immirzi, Class. Quant. Grav. 11, 1971 (1994) [gr-qc/9402004]; Class. Quant. Grav. 13, 2385 (1996) [gr-qc/9512040]; Nucl. Phys. Proc. Suppl. 57, 65 (1997) [gr-qc/9701052].

[37] J. B. Hartle, W. A. Miller and R. M. Williams, Class. Quant. Grav. 14, 2137 (1997) [grqc/9609028].

[38] H. W. Hamber and R. M. Williams, Nucl. Phys. B487 345 (1997); B451 305 (1995); Phys. Rev. D59 064014 (1999).

[39] H. Coxeter, 'Regular Polytopes', Methuen and Co. Ltd., London, 1948.

[40] H. Coxeter, 'Regular Complex Polytopes', Cambridge University press, 1974.

[41] P.S. Alexandroff, 'Combinatorial Topology', Graylock Press, Rochester 1956.

[42] A.D. Alexandroff, 'Konvexe Polyeder', Akademie Verlag, Berlin 1958.

[43] W. Thurston, The Geometry and Topolgy of Three-Manifolds, Princeton University Press (1978); MSRI March 2002 (http://www.msri.org/publications/books/gt3m/PDF/index.pdf).

[44] L. Kirby, Problems in Low -Dimensional Topology, UC Berkley preprint Dec. 1995 (unpublished).

[45] N. Warner, Proc. Roy. Soc. A383 (1982) 359.

[46] J. Cheeger, J. Diff. Geom. 18 (1983) 575. 
[47] L. Castellani, R. Catenacci, M. Debernardi and C. Pagani, Int. J. Mod. Phys. A 19, 1961 (2004) [math-ph/0211008].

[48] F. Archer and R. M. Williams, Phys. Lett. B 273, 438 (1991).

[49] J. W. Barrett, M. Rocek and R. M. Williams, Class. Quant. Grav. 16, 1373 (1999) [grqc/9710056].

[50] J. W. Barrett and R. M. Williams, Class. Quant. Grav. 5, 1543 (1988).

[51] G. Arcioni, M. Carfora, A. Marzuoli and M. O'Loughlin, Nucl. Phys. B 619, 690 (2001) [hep-th/0107112].

[52] M. Carfora, C. Dappiaggi and A. Marzuoli, gr-qc/0310039.

[53] V. M. Khatsymovsky, Phys. Lett. B 586, 411 (2004); ibid. B 567, 288 (2003); B 560, 245 (2003); B 530, 251 (2002).

[54] G. Batrouni, Nucl. Phys. B208 (1982) 467.

[55] G. Batrouni and M. B. Halpern, Phys. Rev. D30 (1984) 1782. 